\title{
Comparative studies of global and targeted control of walkway bridge resonant frequencies
}

\author{
Donald S Nyawako ${ }^{1}$ and Paul Reynolds ${ }^{1,2}$ \\ ${ }^{1}$ University of Exeter, Vibration Engineering Section, College of Engineering, Mathematics and \\ Physical Sciences, Exeter, EX4 4QF, UK \\ ${ }^{2}$ Full Scale Dynamics Limited, 40 Leavygreave Road, Sheffield, S3 7RD, UK
}

\begin{abstract}
In this paper, three controllers are investigated for active vibration control (AVC) of a pedestrian walkway structure. They comprise of direct velocity feedback (DVF), observer-based and independent modal space (IMSC) controllers that are implemented in single-input single-output (SISO), multi-SISO and multiple-input multiple-output (MIMO) configurations. The objective of the SISO controller schemes is to compare vibration mitigation performances arising from global control versus selective control of structural resonant frequencies in a given frequency bandwidth. The objectives set out for the multi-SISO and MIMO controllers are to realise global control within the same frequency bandwidth considered in the SISO studies. A novel aspect of these latter studies is the independent control of selected resonant frequencies at different locations on the structure with the aim of imposing global control.
\end{abstract}

Vibration mitigation performances are evaluated using frequency response function measurements and uncontrolled and controlled responses to a synthesized walking excitation force. In the SISO studies, selective control of specific resonant frequencies has a slight degradation in the global vibration mitigation performance although it reflects better performance around the target frequencies. For the multi-SISO and MIMO controller studies, the selective control of the two lowest and dominant frequencies of the structure at two different locations still offers comparative vibration mitigation performances with the controllers considered as global in the sense that they target both structural frequencies at both locations. Attenuations of between 10-35 dB are achieved.

Keywords: Vibration control, dynamic controllers, velocity feedback, experimental modal analysis, IMSC

\footnotetext{
${ }^{1}$ University of Exeter, Vibration Engineering Section, College of Engineering, Mathematics and Physical Sciences, Exeter, EX4 4QF, UK

2 Full Scale Dynamics Limited, The Sheffield Bioincubator, 40 Leavygreave Road, Sheffield, S3 7RD, UK

Corresponding author:

Donald S Nyawako, University of Exeter, Vibration Engineering Section, College of Engineering, Mathematics and Physical Sciences, Exeter, EX4 4QF, UK Email: D.S.Nyawako@exeter.ac.uk
} 


\section{Introduction}

Increasingly flexible civil engineering structures arise due to advances in material and construction technologies, coupled with client and architectural requirements for more aesthetically appealing structures. The consequences of these factors are more slender, lightweight, and open-plan layouts (Dallard et al., 2001; Falati, 1999; Hanagan and Kim, 2005; Sandun De Silva and Thambiratnam, 2009), that are characterised by low frequencies and modal damping ratios. Their dynamic responses are often found to be unacceptable under human loading, and often fail to satisfy requirements in design guidelines, for example, BD37/01 for footbridges, and BS6472:2008, ISO 10137:2007 and Hicks et al., 2011 for floors. Remedial measures used to enhance the vibration serviceability performance of such facilities might comprise of either passive, active, semi-active or hybrid technologies. These techniques have pros and cons relating to costs (installation, running, disruption etc.), weight constraints and potential vibration mitigation performance that can be achieved.

Research on the impact of using active vibration control (AVC) technology towards floor and footbridge vibration control can be seen in some recent trials (Díaz \& Reynolds, 2010; Hanagan \& Murray, 1998; Nyawako et al., 2013; Casado et al., 2013; Moutinho et al., 2007). These have demonstrated improved vibration mitigation performance in the structures in which AVC has been implemented ranging from $30-80 \%$ in comparison with the uncontrolled responses. The motivation for these studies and the continual development of this technology is that the vibration serviceability performances of such structures under human excitation can be improved significantly whilst allowing them to be more slender, open-plan and lightweight. As well as the potential of being used as retrofits in problematic structures, there is also the possibility for incorporation of AVC during the design phase of future structures. Some challenges in the form of high initial installation and running costs need to be overcome if this technology is to be considered as an economical solution.

The most widely used control strategy in the above field trials is direct velocity feedback (DVF). Measured velocity responses are multiplied by control gains and fed back to collocated actuators to provide global damping in the observable vibration modes. It has been shown by Balas (1978) that provided sensors are collocated with the actuators, the single-input single-output (SISO) and decentralized multiple-input multiple-output (MIMO) systems are bound to be stable with DVF. Apart from the studies noted above for control of human-induced excitation, more rigorous studies to investigate optimal gain features of DVF as well as its stability properties within SISO, decentralized MIMO and MIMO schemes have also been investigated for flexible elements like plates and sandwich panels for noise and vibration control (González Díaz \& Gardonio, 2007; Rohlfing et al., 2011; Zilletti et al., 2010). The focus of most of these past researches has been on global control of the observable vibration modes.

Model-based controllers, on the other hand, have been extensively investigated and implemented in other sectors, for example, in the mechanical engineering and aerospace industries, control of large space structures, as well as in studies with piezoelectric or piezo-ceramic materials (Houlston et al., 2007; Fang et al., 2003; Resta et al., 2010; Daley et al., 2004; Singh et al., 2003, Bosse et al., 2000). A group of model-based controllers of interest to this research work is observer-based compensators and independent modal space control (IMSC) approaches. With the former, all system states are generally not measurable and therefore state observers are included and incorporated in the pole placement design process. The complete controller is then given as an observer in cascade with the state variable feedback. These controllers have been implemented for some specific applications such 
as improving air and fuel characteristics of spark ignition engines (Choi et al., 1998), control of permanent magnet synchronous motor without mechanical sensors (Sepe et al., 1992) and torsion control of flexible shaft systems (Korondi et al., 1998). There have also been successful implementations in other case studies, for example, in multi-modal control of beam and model frame structures (Sethi et al., 2005; Sethi et al., 2007). Some trials have been carried out in large-scale civil engineering structures (Chung et al., 1998; Wu et al., 1998), but they have hardly been investigated and implemented in floor or footbridge vibration control trials. Some challenges concerning design freedoms that guarantee both overall closed-loop stability and controller stabilities with these approaches have been addressed in some research works (Liu et al., 1998; Liu et al., 2000). The IMSC approach has been successfully implemented in applications, for example, in the marine sector for developing isolation systems that improve the crew and passenger comfort (Daley et al., 2004) as well as in suppression of vibrations in a multi-body nonlinear flexible boom test rig (Resta et al., 2010). These control schemes can be designed for either global control (control of a wide bandwidth of frequencies) or they can be tailored for selective control (focusing control energy on selected resonance frequencies).

In this paper, vibration mitigation performance of three controller schemes is investigated on a walkway bridge structure. They comprise of the direct velocity feedback (DVF) controller, observerbased controllers and an independent modal space controller (IMSC). The DVF and observer-based controllers are implemented in both single-input single-output (SISO) and multi-SISO (decentralised) schemes whilst the IMSC controller is implemented in a multiple-input multiple-output (MIMO) scheme. The primary aim of the controller studies with the SISO schemes is to provide comparative studies between global control and selective control objectives of the observable structural resonances within a selected frequency bandwidth. This frequency bandwidth is considered as that excitable from human walking and its harmonics. DVF is by its nature a global controller whilst three observer-based controllers are designed for both global control and selective control. The fundamental aims of the multi-SISO and MIMO control studies are to investigate the impact of isolation and control of specific structural resonant frequencies simultaneously at different locations on the structure with an objective of imposing global control, which is the key novelty of this work. It is carried out with two of the observer-based controllers designed in the SISO studies as well as with the IMSC controller. Comparative studies are carried out with a multi-SISO DVF controller and one set of observer-based controllers that are designed purely for global control.

\section{Control strategies}

The control schemes that are designed in this work and which are used to evaluate the vibration control objectives outlined above comprise of the DVF controller, observer-based controllers and an IMSC controller. DVF and observer-based controllers are implemented in SISO and multi-SISO set-ups whilst the IMSC controller is implemented in a MIMO set-up. The multi-SISO and MIMO set-ups comprise of two actuator and sensor pairs.

These controllers fall into the categories of direct output feedback (DOFB) and model-based schemes. DOFB strategies, for example, DVF are global controllers in the sense that they target all observable structural resonant frequencies in a given bandwidth whilst model-based approaches, for example, the observer-based controllers can be designed for global or selective control. This work exploits these properties of the controllers to provide comparative studies in the vibration mitigation performances. 


\subsection{Direct velocity feedback control}

The DVF controller primarily augments damping of all observable structural frequencies within the frequency bandwidth considered, and is referred to as a global controller in this work by virtue of this property. Provided the sensor and actuator pairs are collocated, SISO and multi-SISO implementations of the controller are bound to be stable. For the SISO and multi-SISO set-ups in this work, the controllers take the forms of Equations 1a and 1b.

$$
\begin{aligned}
& G_{c}(s)=G_{D V F}(s)=\frac{K_{g i}}{s} \\
& \underline{G_{c}}(s)=\underline{G_{D V F}}(s)=\left[\begin{array}{cc}
\frac{K_{g i}}{s} & 0 \\
0 & \frac{K_{g j}}{s}
\end{array}\right]
\end{aligned}
$$

Where $G_{D V F}(s)$ and $\underline{G_{D V F}}(s)$ represent the DVF controllers for the SISO and multi-SISO cases. $K_{g i}$ and $K_{g j}\left(\frac{V}{m / s}\right)$ are the velocity feedback gains that are designed to provide maximum augmentation in structural damping whilst satisfying specified stability requirements. The subscripts $i$ and $j$ refer to the locations for siting the collocated actuator and sensor pairs, and $s=j \omega$.

\subsection{Observer-based controllers}

These controllers offer more design freedoms in comparison with the DVF controller and are of higher order depending on the number of structural resonant frequencies included in the design. In this research work, they are designed for both selective control and global control. The controllers are designed from Figure 1 (Xue et al., 2007; Liu et al., 2000).

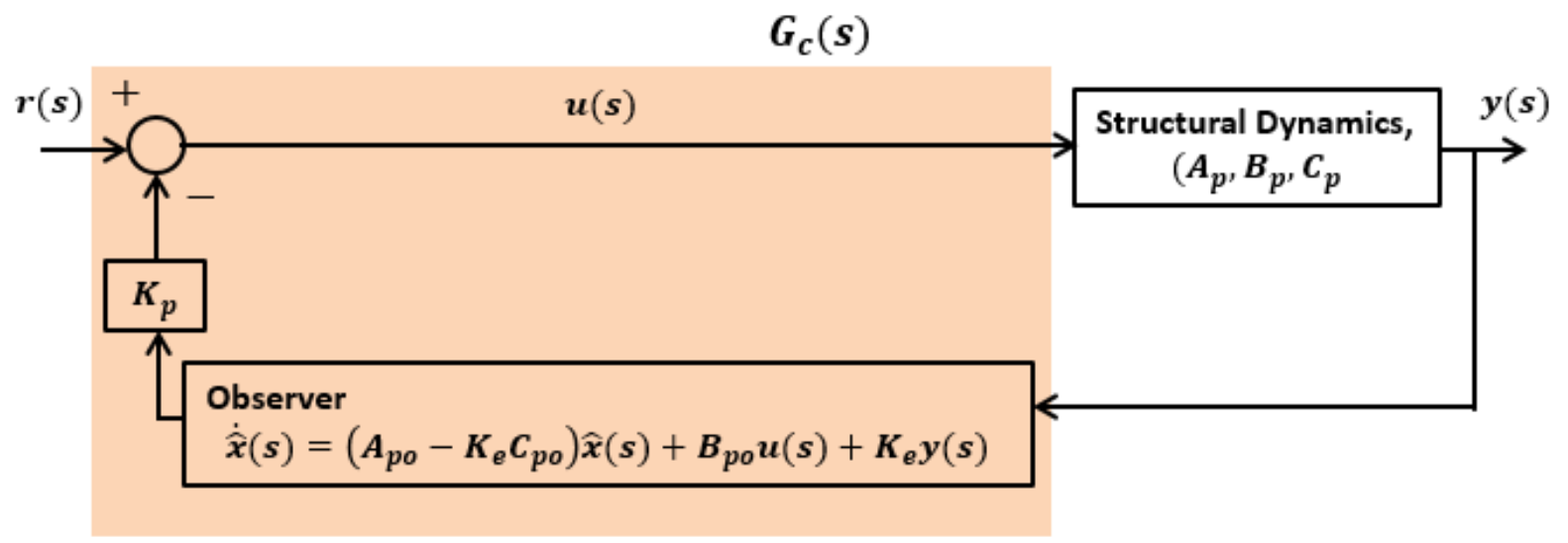

Figure 1. Observer-based compensator

$G_{c}(s)$ is the resulting observer-based controller. $\left(A_{p}, B_{p}, C_{p}\right)$ and $\left(A_{p o}, B_{p o}, C_{p o}\right)$ are state space triples representing the existing structural dynamics and a ROM of the structure that is used in the controller design. $K_{p}$ are feedback gains required to achieve desired closed-loop eigenvalues in the structural dynamics and $K_{e}$ are observer feedback gains. $\hat{x}(s), u(s)$ and $y(s)$ are the estimated modal states of the observer, control input and the structural response.

Following the procedure outlined in Nyawako et al. (2015), the feedback signal, $K_{p} \hat{x}(s)$, is driven by $u(s)$ and $y(s)$ in Equations 2 and 3 in which $\hat{x}_{1}(s)$ and $\hat{x}_{2}(s)$ are only used to distinguish modal states driven by $u(s)$ and $y(s)$. 


$$
\begin{array}{ll}
\dot{\hat{x}}_{1}(s)=\left(A_{p o}-K_{e} C_{p o}\right) \hat{x}_{1}(s)+B_{p o} u(s) & y_{a 1}(s)=K_{p} \hat{x}_{1}(s) \\
\dot{\hat{x}}_{2}(s)=\left(A_{p o}-K_{e} C_{p o}\right) \hat{x}_{2}(s)+K_{e} y(s) & y_{a 2}(s)=K_{p} \hat{x}_{2}(s)
\end{array}
$$

These are then expressed as the two subsystems $H_{u}(s)$ and $H_{y}(s)$ in Equations 4 and 5 in the Laplace domain. The resulting observer-based compensator is shown in Equation 6.

$$
\begin{aligned}
& H_{u}(s)=\frac{Y_{a 1}(s) \cdot \hat{X}_{1}(s)}{\hat{X}_{1}(s) \cdot U(s)}=K_{p}\left(s I-\left(A_{p o}-K_{e} C_{p o}\right)\right)^{-1} B_{p o} \\
& H_{y}(s)=\frac{\hat{X}_{2}(s) \cdot Y_{a 2}(s)}{Y(s) \cdot \hat{X}_{2}(s)}=K_{p}\left(s I-\left(A_{p o}-K_{e} C_{p o}\right)\right)^{-1} K_{e} \\
& G_{c}(s)=G_{o b s_{-} i}(s)=\frac{H_{y}(s)}{1+H_{u}(s)}
\end{aligned}
$$

Three structural resonant frequencies are used in the design in this work resulting in the sixth order controller form in Equation 7. The controllers must be stable and the controllability and observability matrices in Equations 8 and 9 should have full rank.

$$
\begin{aligned}
& G_{c}(s)=G_{\text {obs } \_i}(s)=\frac{k_{T}\left(s+\alpha_{1}\right)\left(s^{2}+\alpha_{2} s+\alpha_{3}\right)\left(s^{2}+\alpha_{4} s+\alpha_{5}\right)}{\left(s^{2}+\beta_{1} s+\beta_{2}\right)\left(s^{2}+\beta_{3} s+\beta_{4}\right)\left(s^{2}+\beta_{5} s+\beta_{6}\right)} \\
& Q_{c}=\left[\begin{array}{lllll}
B_{p o} & A_{p o} B_{p o} & A_{p o}^{2} B_{p o} & \cdots & A_{p o}^{n-1} B_{p o}
\end{array}\right] \\
& Q_{o}=\left[\begin{array}{llll}
C_{p o} & C_{p o} A_{p o} & \cdots & C_{p o} A_{p o}^{n-1}
\end{array}\right]^{T}
\end{aligned}
$$

Where $k_{T}, \alpha_{1}, \ldots, \alpha_{5}$, and $\beta_{1}, \ldots, \beta_{6}$ are the controller coefficients that are designed to achieve various control objectives in terms of either selective control or global control of observable structural resonant frequencies in a selected bandwidth. $Q_{c}$ and $Q_{o}$ are controllability and observability matrices.

The observer-based controllers that are implemented in the SISO and multi-SISO studies are shown in Equations 10a and 10b.

$$
\begin{aligned}
& G_{C}(s)=G_{O B S V}(s)=G_{O b s_{-} i}(s) \\
& \underline{G_{C}}(s)=\underline{G_{O B S V}}(s)=\left[\begin{array}{cc}
G_{O b s_{s} i}(s) & 0 \\
0 & G_{O b s_{-} j}(s)
\end{array}\right]
\end{aligned}
$$

\subsection{Independent modal space control}

The IMSC scheme is used for selective control of structural resonant frequencies of interest and thereby offers additional degrees of freedom compared with the DVF controller. In this work, it is realised via spatial filtering with an array of two discrete actuator and sensor pairs. The discrete modal filters are constructed from orthogonality conditions of the structural mode shapes estimated from experimental modal analysis (EMA) tests. The desired weighting coefficients of the modal filters that isolate the two lowest modes of vibration of the structure considered in this work can be formulated as shown in Equation 11. The concept of spatial filtering is highlighted by Preumont et al. (2003). 
Second order Butterworth low pass filters are introduced to minimize spill-over from higher structural frequencies. The IMSC controller is implemented as a MIMO controller to independently control two of the lowest vibration modes of the structure simultaneously at two different locations with the aim of imposing global control of structural resonant frequencies in a selected frequency bandwidth. This considered as the novelty of this work for comparison with DVF and observer-based controllers.

$$
\underline{G_{c}}(s)=G_{I M S C}(s)=\left[\begin{array}{cc}
\frac{K_{i, i}}{s} & 0 \\
0 & \frac{K_{j, j}}{s}
\end{array}\right]\left\{\left[\begin{array}{cc}
\alpha_{i, i} & \alpha_{i, j} \\
\alpha_{j, i} & \alpha_{j, j}
\end{array}\right]\left[\begin{array}{cc}
G_{p i, i}(s) & G_{p i, j}(s) \\
G_{p j, i}(s) & G_{p j, j}(s)
\end{array}\right]\right\}
$$

$\alpha_{i, i}, \alpha_{i, j}, \alpha_{j, i}, \alpha_{j, j}$ are the linear combiner gain coefficients for selective control and $K_{i, i}, K_{j, j}$ are integral feedback gains that satisfy specified stability requirements. $G_{p i, i}(s)$ and $G_{p j, j}(s)$ are the point accelerance FRFs at selected structural locations for control. $G_{p i, j}(s)$ and $G_{p j, i}(s)$ are the associated cross accelerance FRFs between the same test points.

\section{Controller structure and parameters}

The controller scheme for implementing the controllers above is shown in Figure 2.

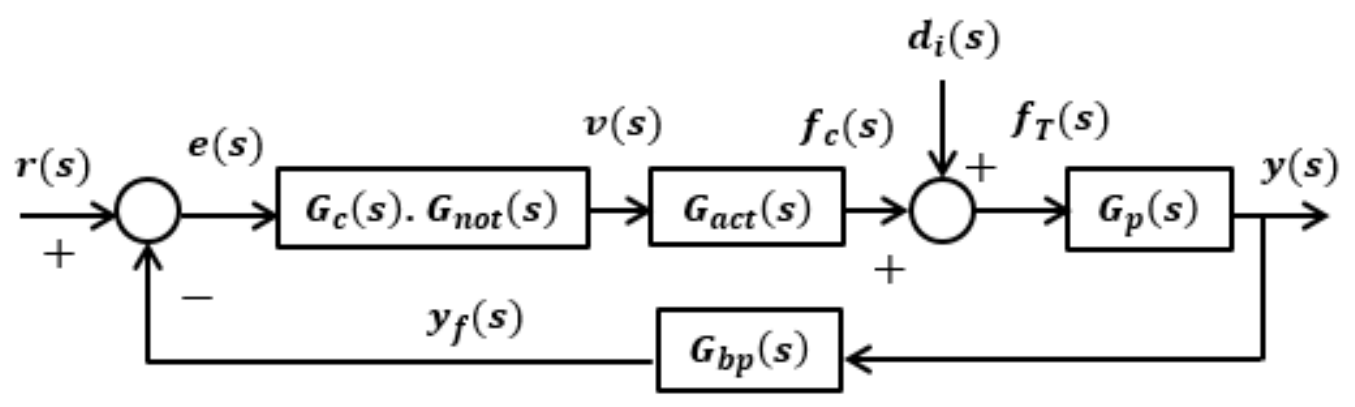

Figure 2. Controller structure for implementing SISO, multi-SISO and MIMO controllers

$G_{p}(s), G_{a c t}(s), G_{b p}(s), G_{n o t}(s)$ and $G_{c}(s)$ represent the structure, actuator, band pass filter, notch filter and controller dynamics for a SISO controller. $d_{i}(s), y(s), f_{c}(s), r(s), e(s)$ and $v(s)$ are the disturbance input, structural acceleration response, actuator force, reference signal, error signal and control signal. For multi-SISO and MIMO controllers, $G_{p}(s), G_{a c t}(s), G_{b p}(s), G_{n o t}(s)$ in Figure 2 can be considered in matrix form as $\underline{G_{p}}(s), \underline{G_{a c t}}(s), \underline{G_{b p}}(s), \underline{G_{n o t}}(s)$, and shown in Equations 12 and 13. $\underline{G_{c}}(s)$ takes the form of Equations $1 \mathrm{~b}, 10 \mathrm{~b}$ and $11 . G_{p}(s)$ is equivalent to the state space triple $\left(A_{p}, B_{p}, C_{p}\right)$ in Figure 1 and the off-diagonal terms in $\underline{G_{p}}(s)$ can be assumed to be zero in the multiSISO studies.

$$
\begin{aligned}
& \underline{G_{p}}(s)=\left[\begin{array}{ll}
G_{p i, i}(s) & G_{p i, j}(s) \\
G_{p j, i}(s) & G_{p j, j}(s)
\end{array}\right], \underline{G_{b p}}(s)=\left[\begin{array}{cc}
G_{b p i, i}(s) & 0 \\
0 & G_{b p j, j}(s)
\end{array}\right] \\
& \underline{G_{a c t}}(s)=\left[\begin{array}{cc}
G_{a c t i, i}(s) & 0 \\
0 & G_{a c t j, j}(s)
\end{array}\right], \underline{G_{n o t}}(s)=\left[\begin{array}{cc}
G_{\text {not } i, i}(s) & 0 \\
0 & G_{n o t} j, j
\end{array}\right],
\end{aligned}
$$




\subsection{Actuator dynamics}

The actuators used in this research work are APS dynamics model 400 electrodynamic shakers shown in Figure 3b. Their force-voltage (N/V) and displacement-voltage $(\mathrm{m} / \mathrm{V})$ characteristics are shown in Equations 14 and 15 (Nyawako et al. 2015). Equation 16 is the notch filter introduced to compensate for the actuator dynamics around its resonant frequency.

$$
\begin{aligned}
& G_{a c t}(s)=\frac{K_{a c t} s^{2}}{s^{2}+2 \zeta_{a c t} \omega_{a c t} s+\omega_{a c t}^{2}} \\
& G_{a c t_{-} d}(s)=\frac{K_{a c t \_d}}{s^{2}+2 \zeta_{a c t} \omega_{a c t} s+\omega_{a c t}^{2}} \\
& G_{n o t}(s)=\frac{s^{2}+2 \zeta_{a c t} \omega_{a c t} s+\omega_{a c t}^{2}}{s^{2}+2 k_{n o t} \zeta_{a c t} \omega_{a c t} s+\omega_{a c t}^{2}}
\end{aligned}
$$

$G_{a c t}(s)$ and $G_{a c t} d(s)$ are the actuator force-voltage and displacement-voltage dynamics and $G_{n o t}(s)$ is a notch filter. $\omega_{\text {act }}=8.17 \mathrm{rad} / \mathrm{s}$ and $\zeta_{\text {act }}=0.10$ are the actuator's resonance frequency and damping ratio. $K_{a c t}=300$ and $K_{a c t \_d}=10$ are constant parameters. $k_{\text {not }}>1$ is the design gain constant of the notch filter for each of the controllers.

\subsection{Structural dynamics}

The structure whose dynamics are used in the studies presented here is a walkway bridge at the Forum

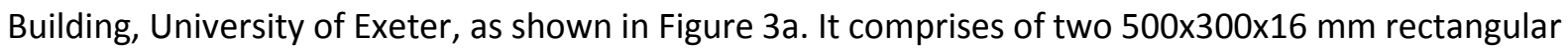
hollow section (RHS) primary beams, $14.5 \mathrm{~m}$ and $14.7 \mathrm{~m}$ in length and spaced at $2.7 \mathrm{~m}$ centre to centre, with tapered $300 \times 200 \times 16 \mathrm{~mm}$ RHS beams welded underneath. Secondary beams of $80 \times 80 \times 8 \mathrm{~mm}$ RHS run every $1800 \mathrm{~mm}$ between and perpendicular to the primary beams. The flooring comprises of a $130 \mathrm{~mm}$ thick concrete on RSLD Holorib S350 profiled metal deck, $0.9 \mathrm{~mm}$ gauge with A193 mesh. Ceramic tiles form the final floor finish. Figure $3 c$ shows the test grid used for EMA tests.

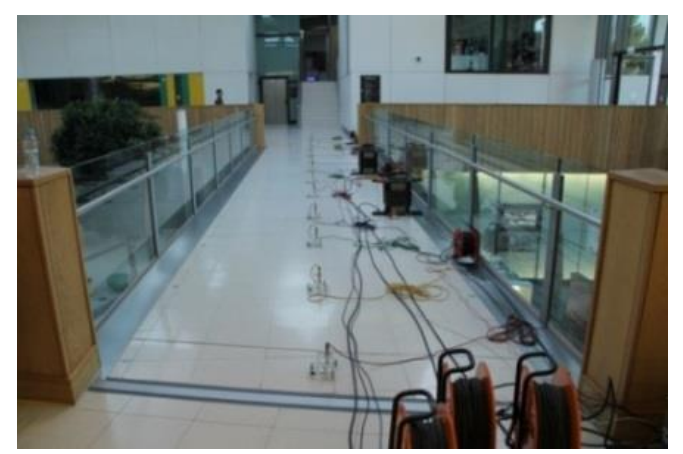

(a) Walkway bridge structure

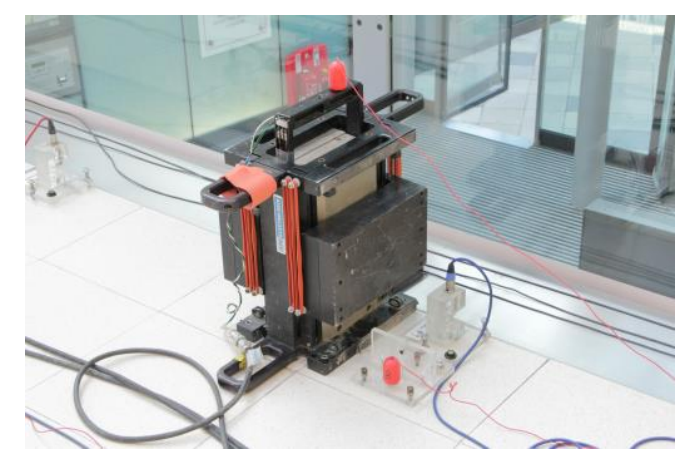

(b) APS actuator 


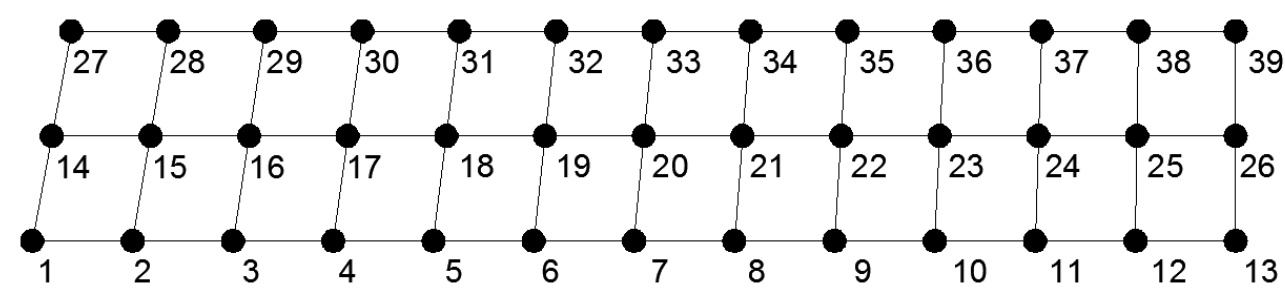

(c) Test grid for EMA tests

Figure 3. Photos of walkway bridge and APS 400 shaker and test grid used for EMA tests

For the EMA tests, two excitation shakers located at TP4 and TP7 were driven by statistically uncorrelated random signals and their forces were measured using two Endevco 7754A-1000 accelerometers attached to their inertial masses. Walkway bridge responses were measured with 13 QA-750 force balance accelerometers that were roved along grid lines 1-13, 14-26 and 27-39. A Data Physics Mobilyzer II digital spectrum analyser was used for data acquisition. Figure 4 shows the point accelerance FRFs at TPs 4 and 7.

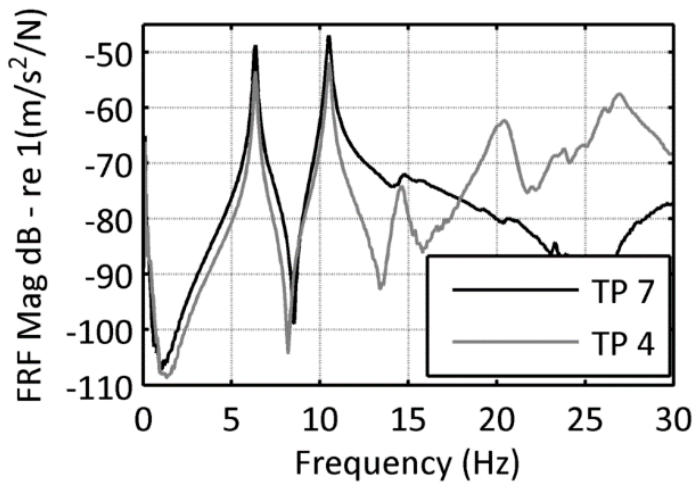

(a)

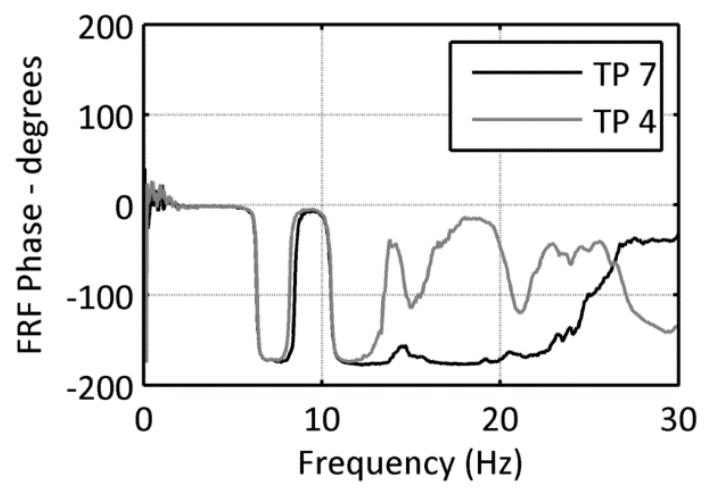

(b)

Figure 4. Magnitude (a) and phase (b) of measured point accelerance FRFs

The FRF data from the EMA was analysed using ME'scopeVES parameter estimation software to determine the modal properties (natural frequencies, modal damping ratios and mode shapes) of the walkway bridge structure. Key numerical results from this estimation process are summarised in Table 1 and mode shapes corresponding with the first two vibration modes are shown in Figure 5.

Table 1. Summary of estimated modal properties from EMA

\begin{tabular}{|c|c|c|}
\hline Mode & Natural Frequency [Hz] & Damping Ratio [\%] \\
\hline 1 & 6.34 & 1.0 \\
\hline 2 & 10.5 & 0.9 \\
\hline 3 & 14.6 & 2.2 \\
\hline 4 & 20.5 & 2.6 \\
\hline 5 & 26.1 & 0.7 \\
\hline 6 & 26.9 & 1.1 \\
\hline 7 & 34.3 & 2.3 \\
\hline
\end{tabular}




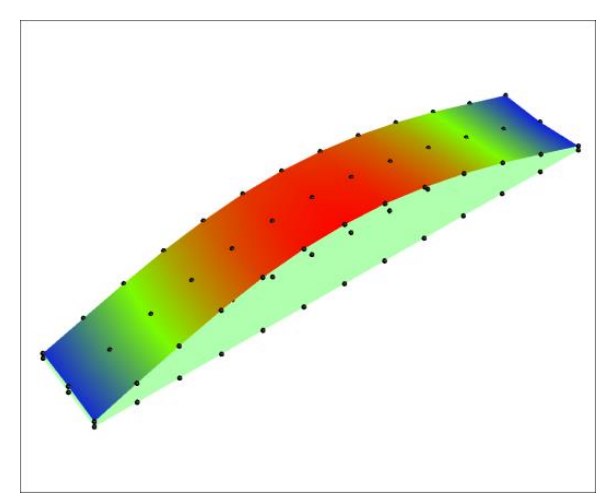

a) Mode shape for mode 1 at $6.34 \mathrm{~Hz}$

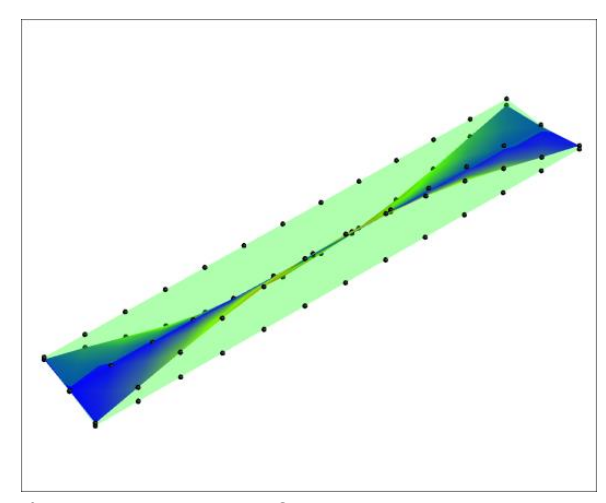

b) Mode shape for mode 2 at $10.5 \mathrm{~Hz}$

Figure 5. Mode shapes corresponding to the lowest two structural resonant frequencies

From the modal estimation procedure, a state space model of the structure can be developed as shown in Equations 17a and 17b. They express the output in terms of modal displacements and velocities. The transformations $\emptyset^{T} x_{1}$ and $\emptyset^{T} x_{2}$ can be used to revert back to the nodal displacements and velocities in the physical space at all sensor locations. Appropriate reduced order models (ROMs) that are required for controller designs and analytical studies can be derived. Equations $17 \mathrm{a}$ and $17 \mathrm{~b}$ can be converted to transfer function form through the transformation in Equation 18.

$$
\begin{aligned}
& \left\{\begin{array}{l}
\dot{x}_{1} \\
\dot{x}_{2}
\end{array}\right\}=\left[\begin{array}{cc}
0 & I \\
-\frac{K^{*}}{M^{*}} & -\frac{C^{*}}{M^{*}}
\end{array}\right]\left\{\begin{array}{l}
x_{1} \\
x_{2}
\end{array}\right\}+\left[\begin{array}{cc}
0 & 0 \\
\emptyset^{T} D & \emptyset^{T} E
\end{array}\right]\left\{\begin{array}{l}
u \\
f
\end{array}\right\}, \dot{x}=A_{p o} x+B_{p o} u \\
& \left\{\begin{array}{l}
y_{1} \\
y_{2}
\end{array}\right\}=\left[\begin{array}{ll}
I & 0 \\
0 & I
\end{array}\right]\left\{\begin{array}{l}
x_{1} \\
x_{2}
\end{array}\right\}+\left[\begin{array}{ll}
0 & 0 \\
0 & 0
\end{array}\right]\left\{\begin{array}{l}
u \\
f
\end{array}\right\}, y=C_{p o} x+D_{p o} u \\
& G_{p}(s)=C_{p o}\left(s I-A_{p o}\right)^{-1} B_{p o}+D_{p o} \\
& K^{*}=\left[\begin{array}{cccc}
\omega_{1}^{2} & 0 & \cdots & 0 \\
0 & \omega_{2}^{2} & \cdots & 0 \\
\vdots & \vdots & \ddots & \vdots \\
0 & 0 & \cdots & \omega_{7}^{2}
\end{array}\right] \quad C^{*}=\left[\begin{array}{cccc}
2 \zeta_{1} \omega_{1} & 0 & \cdots & 0 \\
0 & 2 \zeta_{2} \omega_{2} & \cdots & 0 \\
\vdots & \vdots & \ddots & \vdots \\
0 & 0 & \cdots & 2 \zeta_{7} \omega_{7}
\end{array}\right] \\
& M^{*}=\left[\begin{array}{cccc}
1 & 0 & \cdots & 0 \\
0 & 1 & \cdots & 0 \\
\vdots & \vdots & \ddots & \vdots \\
0 & 0 & \cdots & 1
\end{array}\right] \quad \emptyset=\left[\begin{array}{cccc}
\emptyset_{1,1} & \emptyset_{1,2} & \cdots & \emptyset_{1,7} \\
\emptyset_{2,1} & \emptyset_{2,2} & \cdots & \emptyset_{2,7} \\
\vdots & \vdots & \ddots & \vdots \\
\emptyset_{39,1} & \emptyset_{39,2} & \cdots & \emptyset_{39,7}
\end{array}\right]
\end{aligned}
$$

$M^{*}, C^{*}$ and $K^{*}$ are the $n \times n$ modal mass, damping and stiffness matrices. $D$ is the $m \times m$ actuator location matrix and $E$ is the $m \times m$ excitation force location matrix. $\emptyset$ represents the $m \times n$ mass normalised modal transformation matrix and $\zeta_{k}$ and $\omega_{k}$ are the modal damping ratio and circular natural frequency of the $k^{\text {th }}$ vibration mode. $x_{1}$ and $x_{2}$ represent modal displacements and velocities.

Based on Equations 17 and 18, Figure 6 shows the magnitude and phase characteristics of the derived ROMs for the SISO, multi-SISO and MIMO studies. TP7 $(i=7)$ is selected for siting the collocated sensor and actuator pair for the SISO studies. TPs $7(i=7)$ and $33(j=33)$ are selected for siting the collocated sensor and actuator pairs for the multi-SISO and MIMO studies. The structural resonant 
frequencies defined by modes 1, 2 and 7 in Table 1 are observable at these locations. The vibration modes 1 and 2 fall within the frequency range excitable from the harmonics of walking.

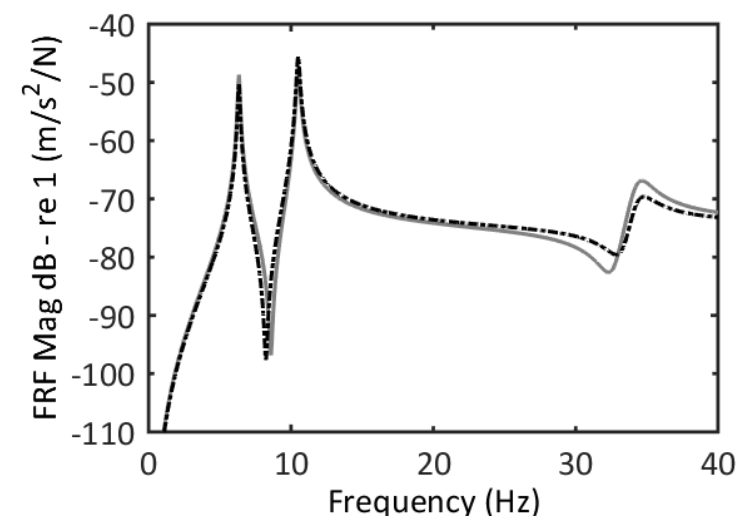

a) FRF magnitude

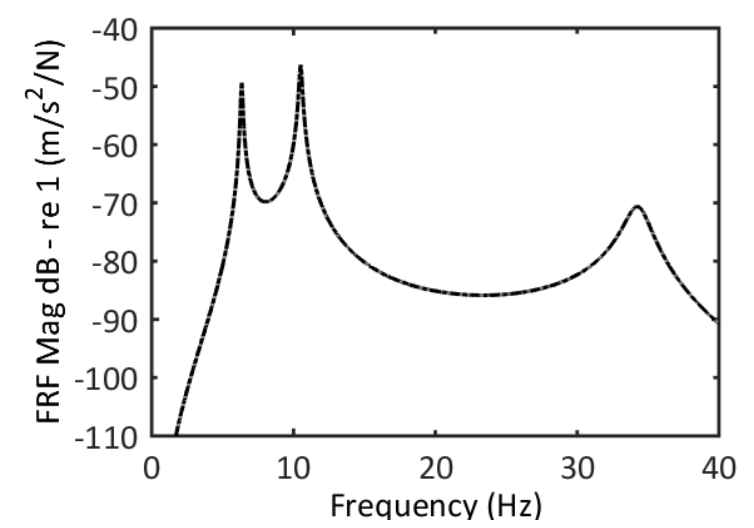

c) FRF magnitude

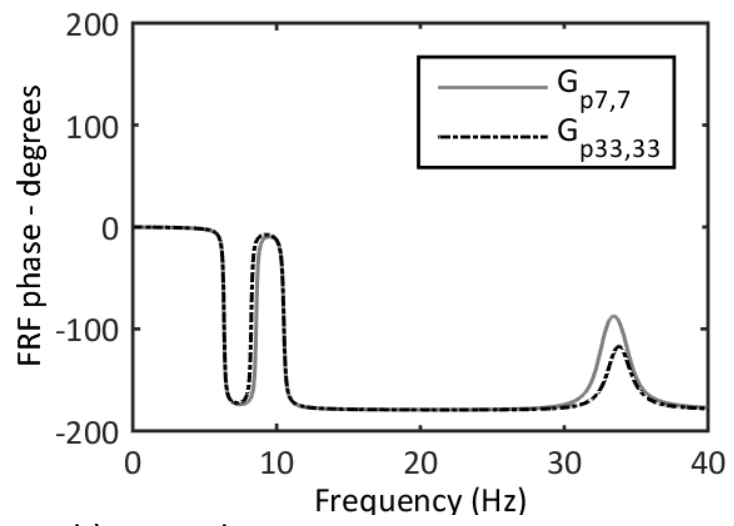

b) FRF phase

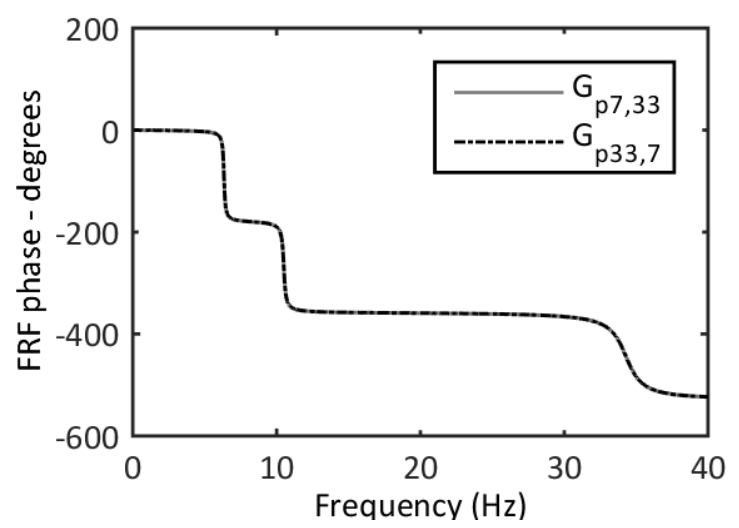

d) FRF phase

Figure 6. Point accelerance FRF magnitudes and phases and cross transfer functions of derived ROMs at TP7 and TP33 for controller design and analytical simulations

\section{Controller parameters}

For mitigation of human-induced vibrations, which is the main focus of this work, disturbance rejection is the primary objective as human walking forces cannot be directly measured. This is evaluated from Equation 19 for SISO control and Equation 20 for multi-SISO and MIMO control.

$$
\begin{aligned}
& Y(s)=\frac{G_{p}(s)}{1+G_{p}(s) G_{n o t}(s) G_{a c t}(s) G_{c}(s) G_{b p}(s)} D_{i}(s) \\
& \underline{Y}(s)=\left(I+\underline{G_{T}}(s)\right)^{-1} \underline{G_{p}}(s) \underline{D_{i}}(s)
\end{aligned}
$$

where $\underline{G_{T}}(s)=\underline{G_{a c t}}(s) \underline{G_{c}}(s) \underline{G_{n o t}}(s) \underline{G_{b p}}(s) \underline{G_{p}}(s)$.

Additional relationships between the actuator displacements to disturbance loops are also derived as shown in Equation 21 for SISO control and Equation 22 for multi-SISO and MIMO control. These are used to provide a constraint on the actuator displacement around its resonance frequency by tuning 
the notch filter compensation in Equation 16 and thereby mitigating the possibility of stroke saturation.

$$
\begin{aligned}
& Y_{d}(s)=\frac{G_{n o t}(s) G_{a c t_{-} d}(s) G_{c}(s) G_{b p}(s) G_{p}(s)}{1+G_{\text {not }}(s) G_{a c t_{-} d}(s) G_{c}(s) G_{b p}(s) G_{p}(s)} D_{i}(s) \\
& \underline{Y_{d}}(s)=\left(I+\underline{G_{U}}(s)\right)^{-1} \underline{G_{U}}(s) \underline{D_{i}}(s)
\end{aligned}
$$

where $\underline{G_{U}}(s)=\underline{G_{a c t \_d}}(s) \underline{G_{c}}(s) \underline{G_{n o t}}(s) \underline{G_{b p}}(s) \underline{G_{p}}(s)$.

The controllers in Equations 1, 10 and 11 are designed to provide maximum augmentation in damping of selected vibration modes of the walkway structure in Equations 19 and 20 whilst meeting the following requirements:

1. Minimum stability margins specified by a Gain Margin (GM) of $5 \mathrm{~dB}$ and Phase Margin (PM) of 30 degrees.

2. The peaks of the actuator displacements to disturbance input relationships in Equations 21 and 22 around the actuator resonant frequency, i.e. $s=j \omega_{\text {act }}$ should not exceed a limit of $0.05 \mathrm{~mm} / \mathrm{N}$.

\subsection{DVF controllers}

In the SISO design, gains of $K_{g i}=K_{g 7}=1150$ and $K_{g i}=K_{g 7}=800$, respectively satisfy the stability margin and actuator stroke to disturbance input limit requirements. $i=7$ denotes the implementation of this controller at TP7 in Figure 3c. The minimum of the two gains, i.e. $K_{g i}=K_{g 7}=$ 800 is the maximum gain implemented with the DVF controller. A second order Butterworth bandpass filter with cut-off frequency $1.0-30.0 \mathrm{~Hz}$ is implemented with this controller to eliminate sensor DC offset as well as filter out high frequency noise. The notch filter constant is designed as $k_{\text {not }}=12$.

For the multi-SISO implementation of the DVF controller at TP7 and TP33 in Figure 3c, i.e. $i=7$ and $j=33$ in Equation $1 \mathrm{~b}$, again the governing factor for the feedback gains is the actuator displacement to disturbance input relationship in Equation 22. This results in feedback gains of $K_{g i}=K_{g j}=$ 850 that are implemented at both control locations.

\subsection{Observer-based controllers}

In the SISO designs, three observer-based compensators are designed to realise the following objectives:

1. Controller $1\left(G_{o b s_{-} i}=G_{c 1}\right)$ is designed for selective control of the first vibration mode

2. Controller $2\left(G_{o b s_{-} i}=G_{c 2}\right)$ is designed for selective control of the second vibration mode

3. Controller $3\left(G_{o b s_{-} i}=G_{c 3}\right)$ is designed to control both the first and second vibration modes

Table 2 shows the open-loop eigenvalues of the three structural resonant frequencies that are observable at TP7. An iterative procedure is adopted to design the desired closed-loop eigenvalues of these structural resonant frequencies that meet the objectives above and these are also shown within Table 2. The resultant controllers in Equations 23, 24 and 25 are stable and the controllability and 
observability matrices in Equations 8 and 9 have full rank. Through the implementation of the controllers in Figure 2, the resultant closed-loop eigenvalues of the structural resonant frequencies are also shown in Table 2. Second order Butterworth band pass filters with cut-off frequencies of 0.5 $-25.0 \mathrm{~Hz}, 0.5-30.0 \mathrm{~Hz}$ and $0.5-40.0 \mathrm{~Hz}$, respectively, are implemented with each of the controllers $G_{c 1}, G_{c 2}$ and $G_{c 3}$. As noted above, their purpose is to eliminate DC offsets in sensor measurements as well as to filter out high frequency noise. The controllers satisfy the actuator displacement to disturbance input limit. The notch filter constant is designed as $k_{n o t}=5$.

$$
\begin{aligned}
& G_{c 1}(s)=\frac{153.5(s+596.5)\left(s^{2}+2.9 s+4339\right)\left(s^{2}+89.2 s+2.6 e 4\right)}{\left(s^{2}+71 s+2161\right)\left(s^{2}+10.2 s+4266\right)\left(s^{2}+39.6 s+4.6 e 4\right)} \\
& G_{c 2}(s)=\frac{349.2(s+969.2)\left(s^{2}+0.8 s+1582\right)\left(s^{2}+24.7 s+4.5 e 4\right)}{\left(s^{2}+189.5 s+8771\right)\left(s^{2}+9.6 s+1665\right)\left(s^{2}+31.2 s+4.6 e 4\right)} \\
& G_{c 3}(s)=\frac{463.7(s+244.1)\left(s^{2}+6.3 s+2706\right)\left(s^{2}+11.3 s+4.7 e 4\right)}{\left(s^{2}+80.9 s+2392\right)\left(s^{2}+20.3 s+2893\right)\left(s^{2}+11.2 s+4.6 e 4\right)}
\end{aligned}
$$

Table 2. Desired and achieved walkway bridge closed-loop eigenvalues for SISO schemes

\begin{tabular}{|c|c|c|c|c|}
\hline Controller & & Mode 1 & Mode 2 & Mode 7 \\
\hline & & & & \\
\hline Open-loop poles & & $-0.41 \pm 39.77 \mathrm{i}$ & $-0.61 \pm 65.97 \mathrm{i}$ & $-5.62 \pm 215.44 \mathrm{i}$ \\
\hline & & & & \\
\hline$G_{\text {obs } i}=G_{c 1}$ & Desired & $-6.57 \pm 40.23 \mathrm{i}$ & $-1.22 \pm 65.96 \mathrm{i}$ & $-8.44 \pm 215.35 \mathrm{i}$ \\
\hline & Achieved & $-6.15 \pm 39.77 \mathrm{i}$ & $-1.38 \pm 65.47 \mathrm{i}$ & $-6.62 \pm 215.44 \mathrm{i}$ \\
\hline$G_{\text {obs } i}=G_{c 2}$ & Desired & $-0.83 \pm 39.76 \mathrm{i}$ & $-9.19 \pm 61.33 \mathrm{i}$ & $-7.03 \pm 215.40 \mathrm{i}$ \\
\hline & Achieved & $-0.41 \pm 39.77 \mathrm{i}$ & $-7.82 \pm 61.20 \mathrm{i}$ & $-5.62 \pm 215.44 \mathrm{i}$ \\
\hline$G_{\text {obs } i}=G_{c 3}$ & Desired & $-4.34 \pm 41.53 \mathrm{i}$ & $-5.63 \pm 63.75 \mathrm{i}$ & $-5.62 \pm 215.44 \mathrm{i}$ \\
\hline & Achieved & $-4.15 \pm 44.64 \mathrm{i}$ & $-3.86 \pm 59.65 \mathrm{i}$ & $-5.62 \pm 215.44 \mathrm{i}$ \\
\hline
\end{tabular}

For the multi-SISO studies, two controller set combinations are considered as follows:

1. $G_{o b s_{-} i}=G_{c 1}$ and $G_{o b s_{-} j}=G_{c 2}$ in Equation $10 \mathrm{~b}$. The aim of this study is to impose a global control objective by independently controlling the two lowest resonant frequencies of the structure at two different locations.

2. $G_{o b s_{-} i}=G_{c 3}$ and $G_{o b s_{-} j}=G_{c 3}$ in Equation $10 \mathrm{~b}$.

Both controller combinations satisfy the actuator stroke to disturbance limit.

\subsection{IMSC controller}

The linear combiner coefficients in Equation 11 are selected as $\alpha_{7,7}=1, \alpha_{7,33}=1, \alpha_{33,7}=1$ and $\alpha_{33,33}=-1$. The designed velocity feedback gain parameters are $K_{i, i}=K_{7,7}=875$ and $K_{j, j}=$ $K_{33,33}=1500$. Together with the linear combiner coefficients, these velocity gains isolate and control modes 1 and 2 at TPs 7 and 33, respectively, with this MIMO control set-up. The primary objective of this study is to impose global control by isolating and simultaneously controlling the lowest two resonant frequencies of the structure for comparative studies with DVF and observer-based compensators. Second order Butterworth band pass filters with the cut-off frequencies of $0.75-20.0$ $\mathrm{Hz}$ are implemented with this controller set. The designed gains satisfy the actuator stroke to 
disturbance limit and minimum stability margin requirements. With this design approach, it is seen that marginally higher feedback gains can be implemented which satisfy the stability requirements.

\section{Stability studies}

Stability of SISO controllers is evaluated via the Nyquist contour plots of $G_{c}(s) G_{a c t}(s) G_{n o t}(s) G_{b p}(s) G_{p}(s)$, which are highlighted in Figure 7 . These do not encircle the $-1,0$ point which shows the stability of all the controllers.

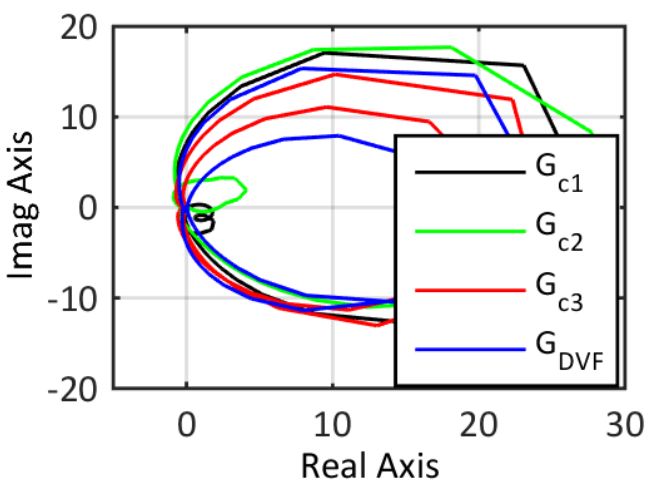

a) Entire contour plot

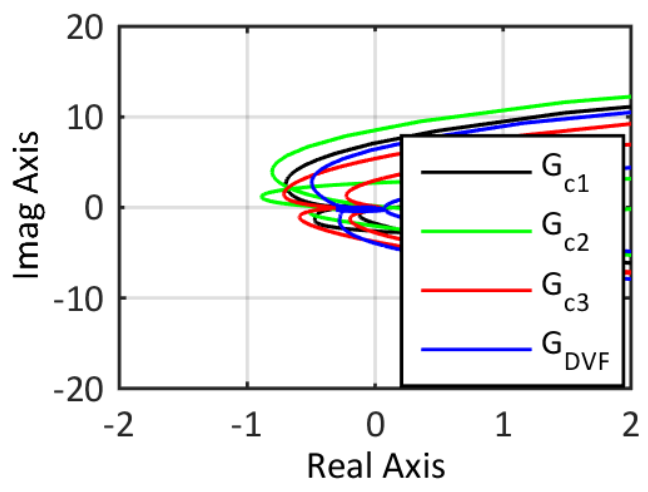

b) Zoomed into plot

Figure 7. Nyquist contour plots of $G_{c}(s) G_{a c t}(s) G_{n o t}(s) G_{b p}(s) G_{p}(s)$ for DVF and observer-based controllers in SISO studies

Stability of the multi-SISO (DVF and observer-based) and MIMO (IMSC) controllers is evaluated from a plot of the eigenvalues of $\underline{F}(s)$ in Equation 26 (Maciejowski 1989). This is shown in Figure 8. All the eigenvalues are in the left of the complex s-plane.

$$
\underline{F}(s)=I+\underline{G_{T}}(s)
$$

where $\underline{G_{T}}(s)=\underline{G_{a c t}}(s) \underline{G_{c}}(s) \underline{G_{n o t}}(s) \underline{G_{b p}}(s) \underline{G_{p}}(s)$. 


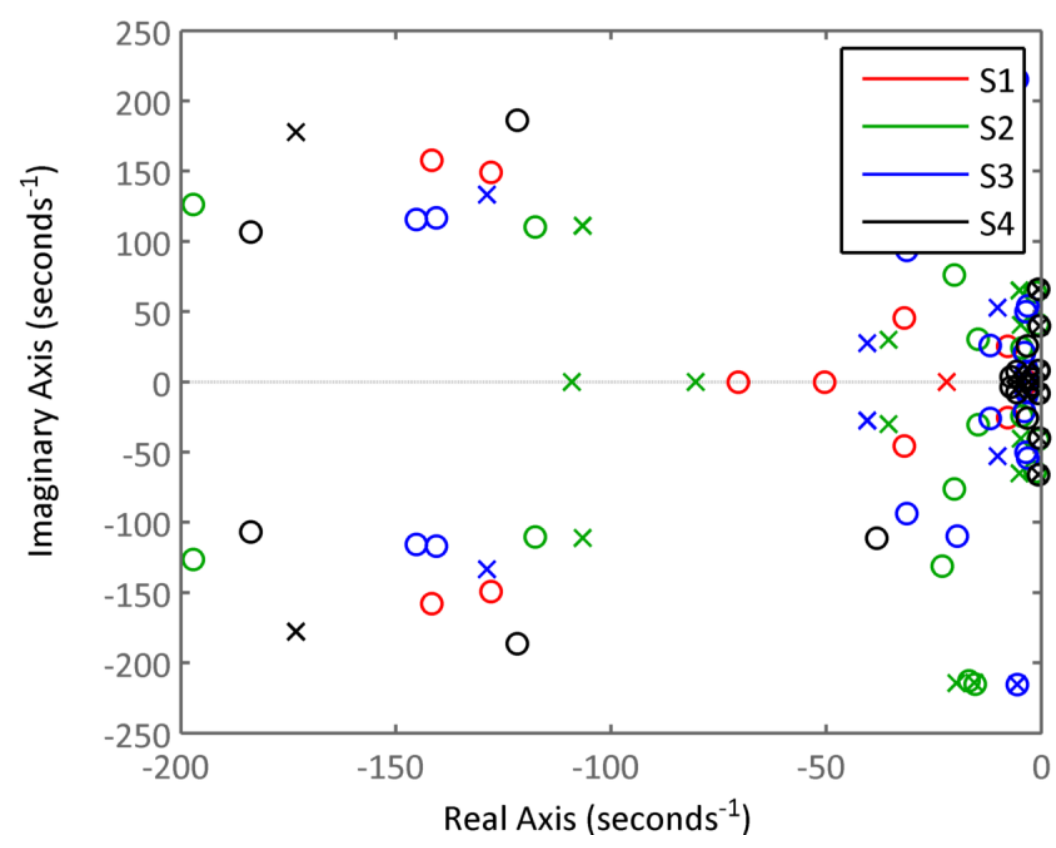

Figure 8. Stability of multi-SISO and MIMO controllers through a plot of the eigenvalues of $\underline{F}(s)$.

S1 refers to the closed-loop system with the DVF controller $\underline{G_{D V F}}(s)$, S2 refers to the closed-loop system with the observer-based compensator $\underline{G_{O B S V}}(s)$ in which $G_{o b s_{i} i}(s)=G_{c 1}(s)$ and $G_{o b s_{-} j}(s)=$ $G_{c 2}(s)$, S3 refers to the closed-loop system with the observer-based compensator $\underline{G_{O B S V}}(s)$ in which $G_{\text {obs } i}(s)=G_{c 3}(s)$ and $G_{o b s_{-} j}(s)=G_{c 3}(s)$ and $S 4$ refers to the closed-loop system with the IMSC controller $\underline{G_{I M S C}}(s)$.

\section{Performance of controlled structure}

The results of the analytical studies presented here comprise of:

a) Uncontrolled and controlled frequency response function (FRF) plots

b) Uncontrolled and controlled responses to a synthesized walking excitation force

\subsection{Uncontrolled and controlled frequency response functions (FRFs)}

These are evaluated from the disturbance rejection properties in Equations 19 and 20. Figures 9a and $9 \mathrm{~b}$ show the FRF magnitudes and phases of the uncontrolled and controlled point accelerance FRFs evaluated at TP7 for the SISO controllers. Figures 10a and 10b also reflect the uncontrolled and controlled FRF magnitudes and phases for the multi-SISO and MIMO controllers. The attenuations in the lowest two modes of vibration of the walkway bridge structure are shown in Table 4. Only the controlled FRFs at TP7 are shown here. 


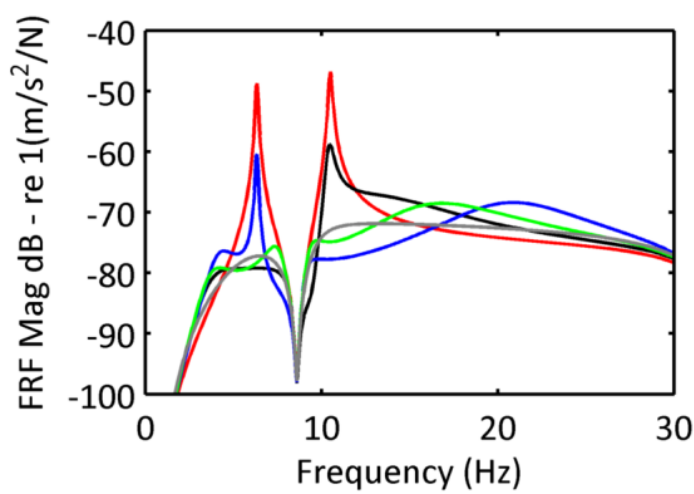

(a) FRF magnitudes

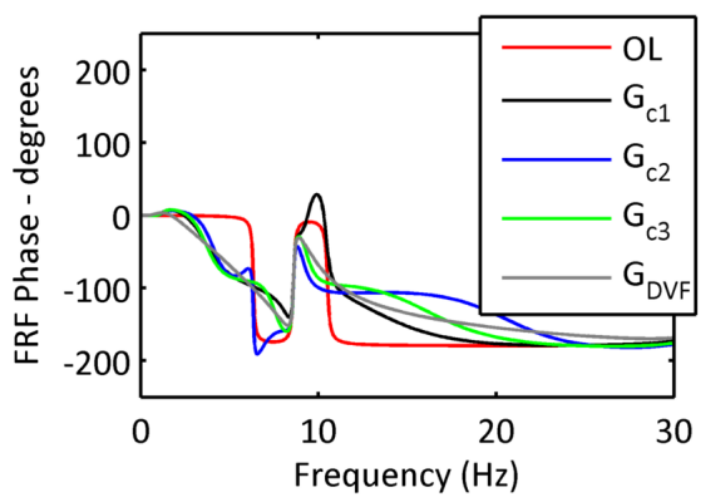

(b) FRF phases

Figure 9. Uncontrolled and controlled FRFs with DVF scheme and observer-based compensators in SISO schemes

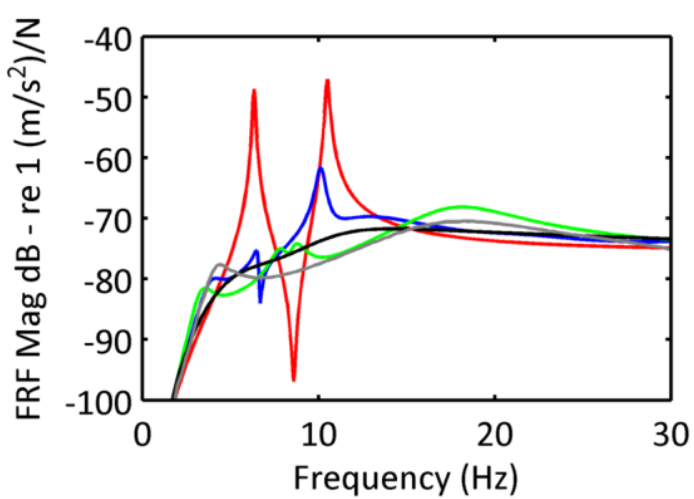

(a) FRF magnitudes

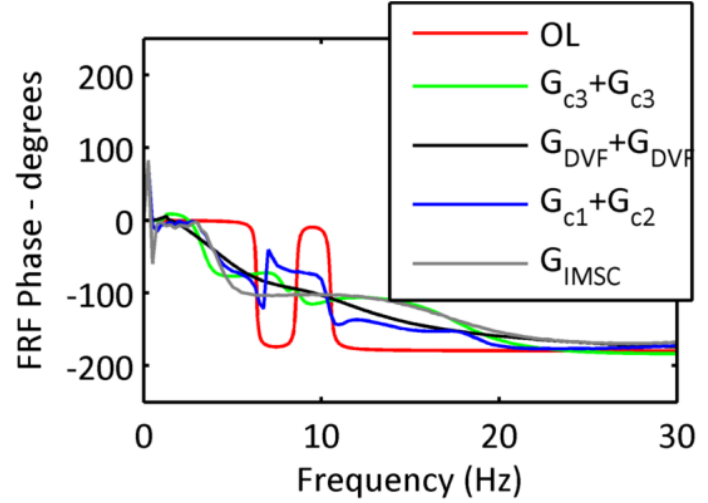

(b) FRF phases

Figure 10. Uncontrolled and controlled FRFs with multi-SISO and MIMO compensators comprising of DVF, observer-based compensators and IMSC controller

Table 4. Attenuations in target modes of vibration for all controllers

\begin{tabular}{|c|c|c|}
\hline Controller & \multicolumn{2}{|c|}{ Predicted attenuations (dB) } \\
\hline & & \\
\hline SISO (TP7) & Mode 1 & Mode 2 \\
\hline$G_{D V F}(s)$ & 28.5 & 25.9 \\
\hline$G_{c 1}(s)$ & 30.5 & 12.0 \\
\hline$G_{c 2}(s)$ & 11.7 & 31.0 \\
\hline$G_{c 3}(s)$ & 29.7 & 28.1 \\
\hline & & \\
\hline Multi-SISO/MIMO (TP7 + TP33) & Mode 1 & Mode 2 \\
\hline$G_{D V F}(s)$ & 29.5 & 26.4 \\
\hline$G_{C 1}(s)+G_{c 2}(s)$ & 26.6 & 14.9 \\
\hline$G_{C 3}(s)+G_{c 3}(s)$ & 32.3 & 29.7 \\
\hline$G_{I M S C}(s)$ & 28.1 & 29.7 \\
\hline
\end{tabular}


The flexibility offered by the observer-based compensators towards selective control as well as global control of observable structural resonant frequencies in a frequency bandwidth considered can be seen in Figure 9. This is in comparison to a purely DVF controller whose inherent feature is to impose a global control objective. All the controllers were found to possess the desirable interlacing property between the structural poles and zeros.

As seen in Figure 10, the multi-SISO DVF controller still shows improved vibration mitigation performance over the frequency bandwidth considered. The implementation of the observer-based controller $G_{c 1}(s)+G_{c 2}(s)$ in a multi-SISO framework at TPs 7 and 33 does not necessarily provide an improvement in the global vibration control objective over the frequency bandwidth considered. This is one of the key objectives in this work that aims to study global vibration mitigation performance by isolating and independently controlling selected structural resonant frequencies at different locations on the structure. The implementation of the observer-based controllers $G_{c 3}(s)+G_{c 3}(s)$ in a multiSISO framework at TPs 7 and 33 offers a desirable global vibration mitigation performance in the frequency bandwidth considered.

Isolation and control of the lowest two resonance frequencies of the structure independently with the IMSC controller in a MIMO scheme shows a desirable vibration mitigation performance. As one of the key objectives and the novelty of this work, this demonstrates that the desire to impose global control with selective control of particular structural resonance frequencies over a considered frequency bandwidth can be achieved. This is realised whilst maintaining appropriate stability requirements.

\subsection{Uncontrolled and controlled responses to synthesized walking excitation}

The synthesized walking excitation force in Figure 11a is used as the disturbance input in the uncontrolled and controlled studies. This is derived by filtering a measured response time history of a pedestrian walking over the walkway bridge structure at a pacing frequency of $2.1 \mathrm{~Hz}$ through the inverse plant dynamics $\left(G_{p i, i}(s)\right)^{-1}=\left(G_{p 7,7}(s)\right)^{-1}$ at TP7. The pacing frequency of the pedestrian was set to excite the lowest two resonance frequencies of the structure; the third harmonic excites the first resonance frequency of the structure and also coincidentally the fifth harmonic excites the second resonance frequency. The Fourier spectrum of the force time history is shown in Figure 11b.

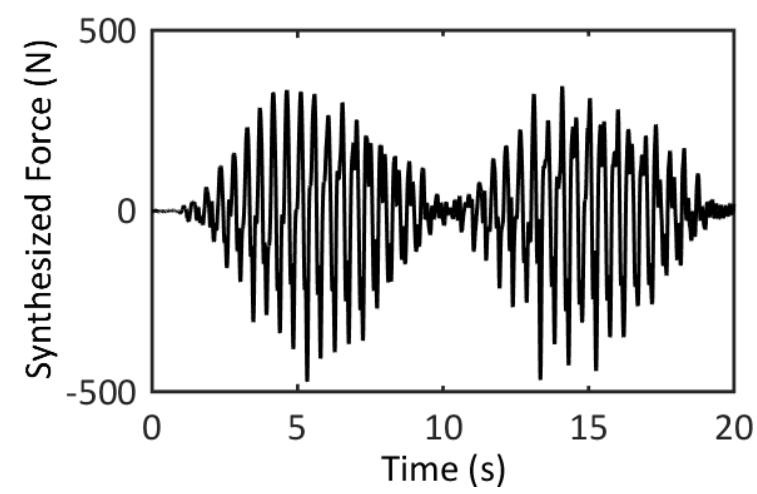

(a) Synthesized walking force

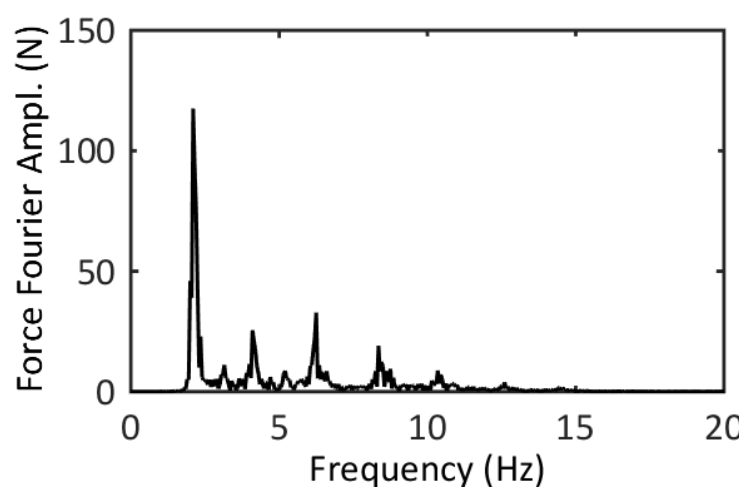

(b) Fourier spectrum of synthesized force

Figure 11. (a) Synthesized walking force and (b) Fourier spectrum of synthesized walking force 
Uncontrolled and controlled responses are monitored at two locations, being TPs 7 and 33 in Figure $3 c$.

In SISO studies, Figure 12 shows uncontrolled and controlled (with DVF controller) responses at TP 7 (responses at TPs 7 and 33 were found to be virtually identical), weighted using the BS6841:1987 Wb weighting function. Vibrations that can be perceived by a person depends on a myriad of factors, for example, direction of incidence to the human body, frequency content of the vibration and duration of the sustained vibrations. The BS6841 Wb weighting function (BS6841, 1987) is applied to response time histories to account for the human sensitivity to vibration at different frequencies.

Figure 13 highlights the Fourier spectra of the uncontrolled and controlled responses at TP 7 only. The Fourier spectra at TPs 7 and 33 are identical and only that at TP 7 is shown here. These are zoomed around $5-12 \mathrm{~Hz}$ and the vertical scales of those of the controlled cases have been adjusted for clarity. They illustrate the effect of the designed controllers in the sense that DVF is a broadband frequency controller, whilst observer-based compensators are tailored for selective control and global control objectives. The magnitudes of the control forces are also shown in Figure 14 for all controllers. The peaks of the $1 \mathrm{~s}$ running root mean square (RMS) acceleration responses are shown in Table 5 . These are defined as the maximum transient vibration value (MTVV) following the recommendation of International Organization of Standardization: ISO 2631 (1997).

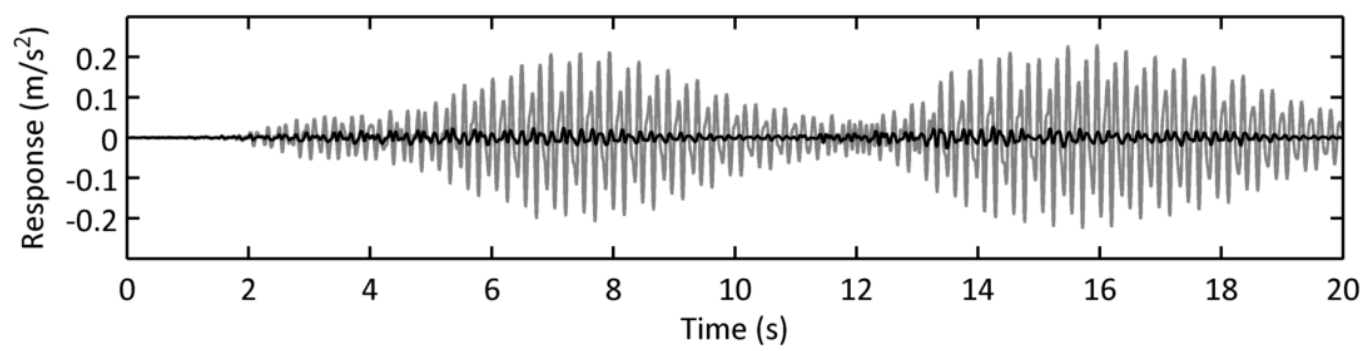

Figure 12. Uncontrolled and controlled responses with DVF controller for SISO control scheme at TP 7 


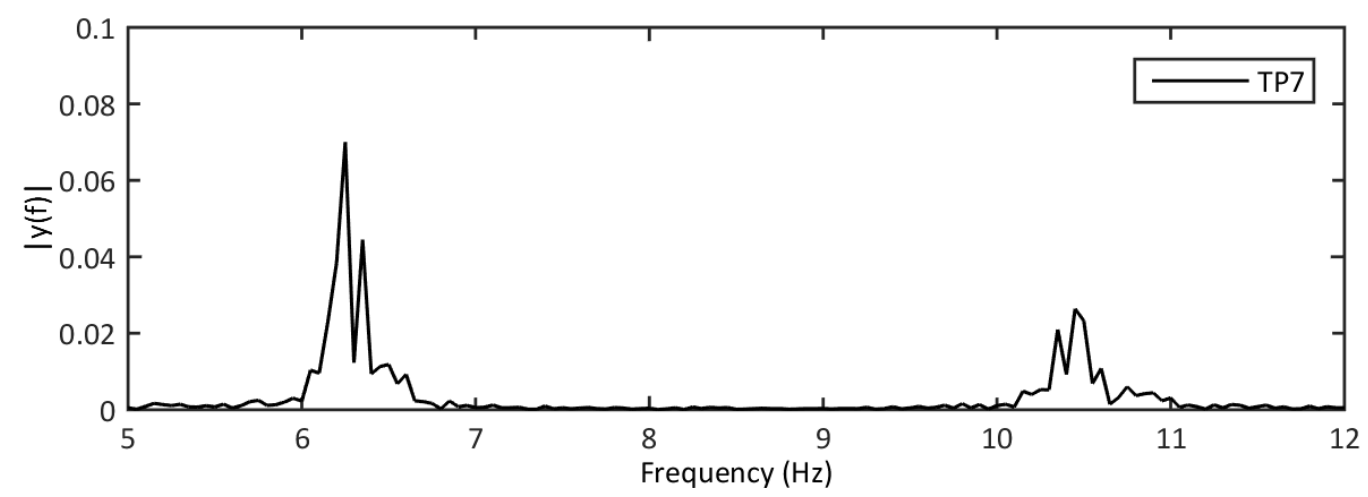

(a) Uncontrolled traces at TPs 7 and 20

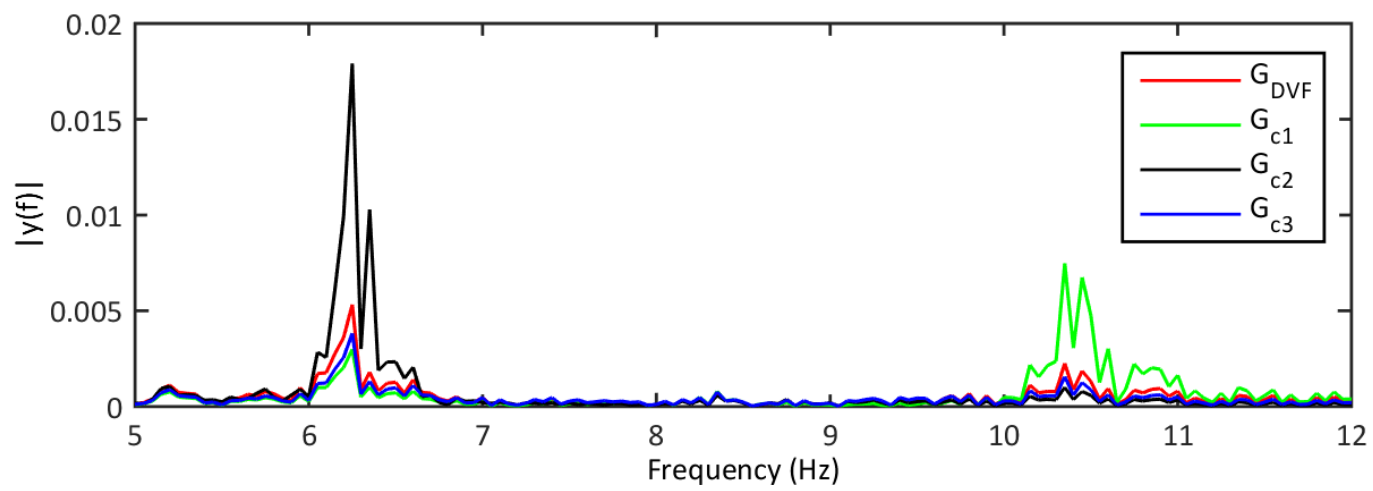

(b) Controlled traces at TP7 with all controllers

Figure 13. Fourier linear spectra of uncontrolled and controlled responses at TPs 7 and 20

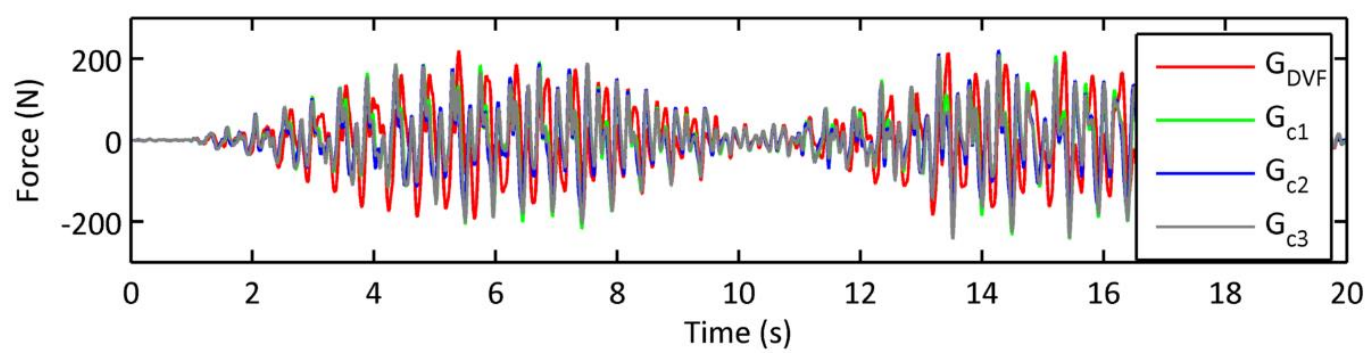

(a) Control forces

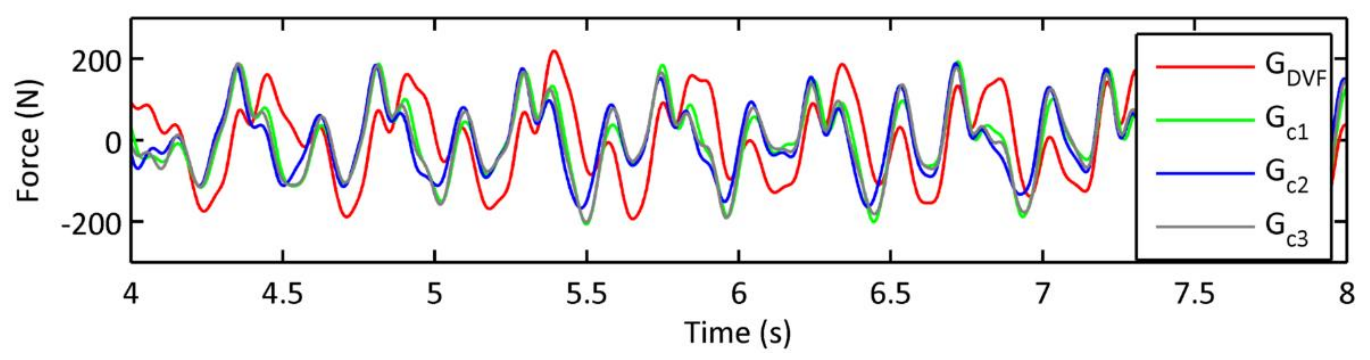

(b) Control forces (zoomed)

Figure 14. (a) Control forces for controllers implemented in the SISO studies - actuator at TP7 (b) Control forces for all controllers in SISO studies - zoomed into plots between $4-8$ seconds 
Table 5. Peak of 1s Running RMS acceleration responses for SISO controller studies

\begin{tabular}{|c|c|c|c|c|}
\hline Controller & \multicolumn{2}{|c|}{$\begin{array}{c}\text { Acceleration at TP7 } \\
\left(\mathbf{m} / \mathbf{s}^{2}\right)\end{array}$} & \multicolumn{2}{c|}{$\begin{array}{c}\text { Acceleration at TP33 } \\
\left(\mathbf{m} / \mathbf{s}^{2}\right)\end{array}$} \\
\hline & & $\%$ reduction & & $\%$ reduction \\
\hline Uncontrolled & 0.1338 & & 0.1259 & \\
\hline$G_{D V F}(s)$ & 0.0134 & 90.0 & 0.0165 & 86.9 \\
\hline$G_{c 1}(s)$ & 0.0174 & 87.0 & 0.0214 & 83.0 \\
\hline$G_{c 2}(s)$ & 0.0317 & 76.3 & 0.0299 & 76.2 \\
\hline$G_{c 3}(s)$ & 0.0105 & 92.1 & 0.0153 & 87.9 \\
\hline
\end{tabular}

Attenuations in structural responses of $76 \%-93 \%$ are seen in the results in Table 5 for all controllers implemented in the SISO studies. The global controllers, i.e. the DVF controller, $G_{D V F}(s)$, and observer-based controller, $G_{c 3}(s)$ offer the best vibration mitigation performance as they attenuate both the first and second resonance frequencies of the structure that are excitable from their subharmonics of walking. There is a slight degradation in the vibration mitigation performances with the observer-based controllers designed primarily for selective control of particular structural resonance frequencies, i.e. $G_{c 1}(s)$ and $G_{c 2}(s)$. They, however, have a greater control impact at the structural resonance frequencies for which they are tuned to as seen in Fourier spectra in Figure 13b. In Figure $14 \mathrm{a}$ and $14 \mathrm{~b}$, there is not much variation in the peak amplitudes of the control forces resulting from all the controller although some phase variations can be seen.

For the multi-SISO DVF controller, Figures $15 \mathrm{a}$ and $15 \mathrm{~b}$ highlight the uncontrolled and controlled responses at TPs 7 and 33. They are also weighted using BS6841:1987 Wb weighting function. The Fourier spectra of the controlled responses at the same test points for all the controllers investigated here are shown in Figure 16. These are also zoomed within the frequency span of interest of $5-12 \mathrm{~Hz}$ and similar vertical scales to those in Figure $13 \mathrm{~b}$ are used. The magnitudes of the control forces for all controllers are also shown in Figure 17 . The peaks of the $1 \mathrm{~s}$ running root mean square (RMS) acceleration responses, weighted using BS6841:1987 Wb weighting function are also shown in Table 6. 


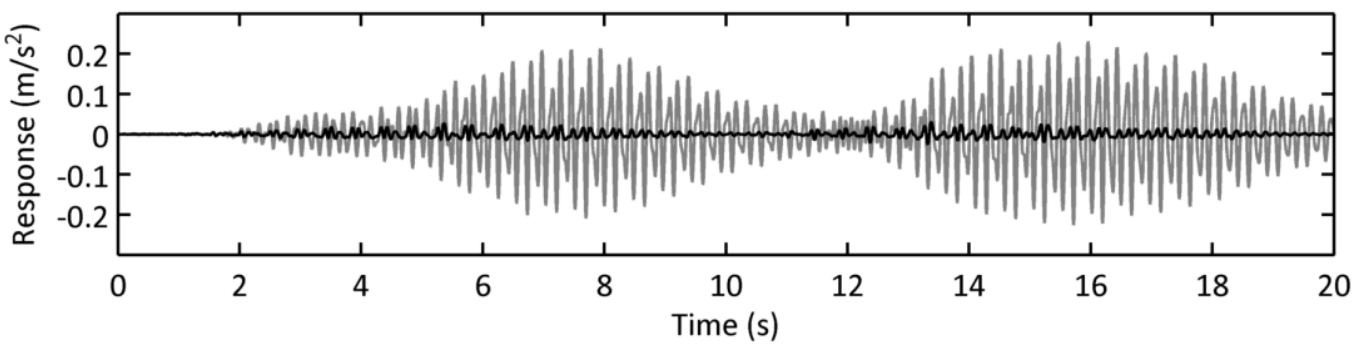

(a) Uncontrolled and controlled response at TP7

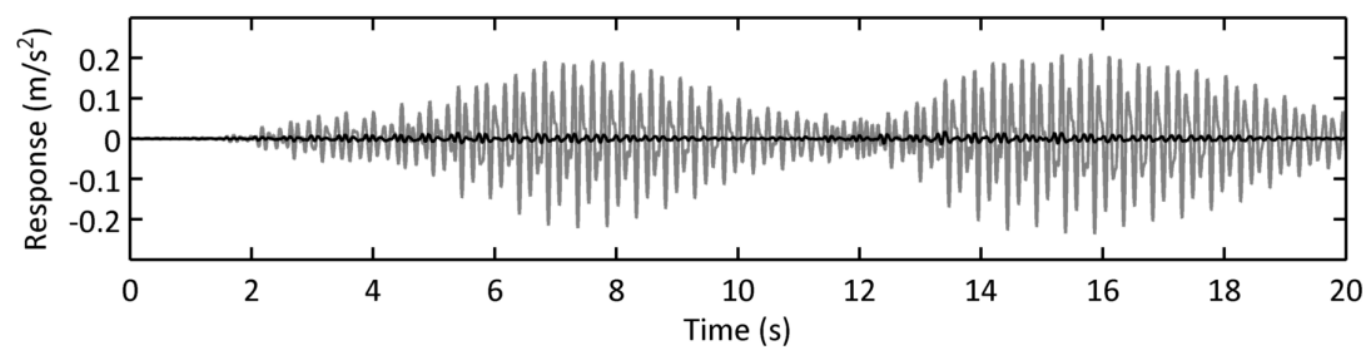

(b) Uncontrolled and controlled response at TP33

Figure 15. Typical uncontrolled and controlled responses with DVF controller at TPs 7 and 33

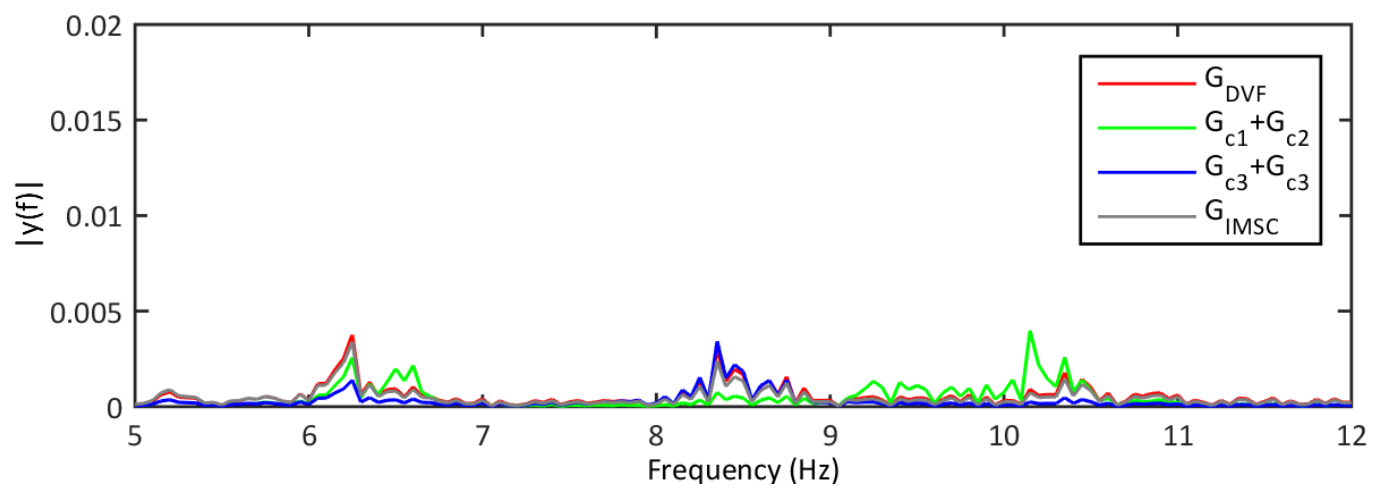

(a) TP7

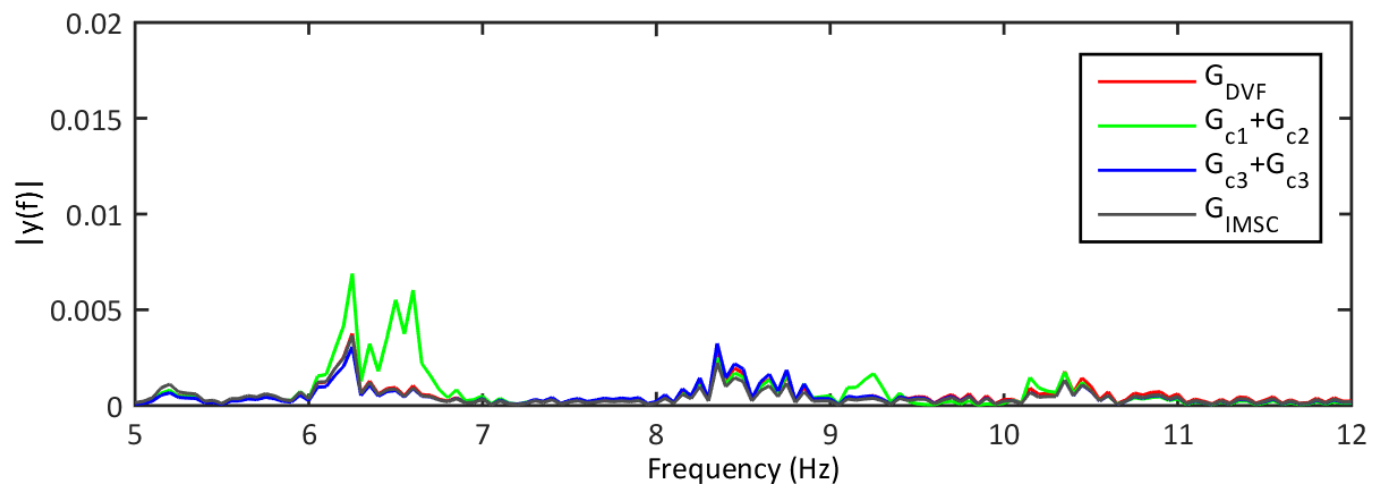

(b) TP33

Figure 16. Fourier spectra of controlled responses at TPs 7 and 33 for all controller sets used in the multi-SISO and MIMO studies 


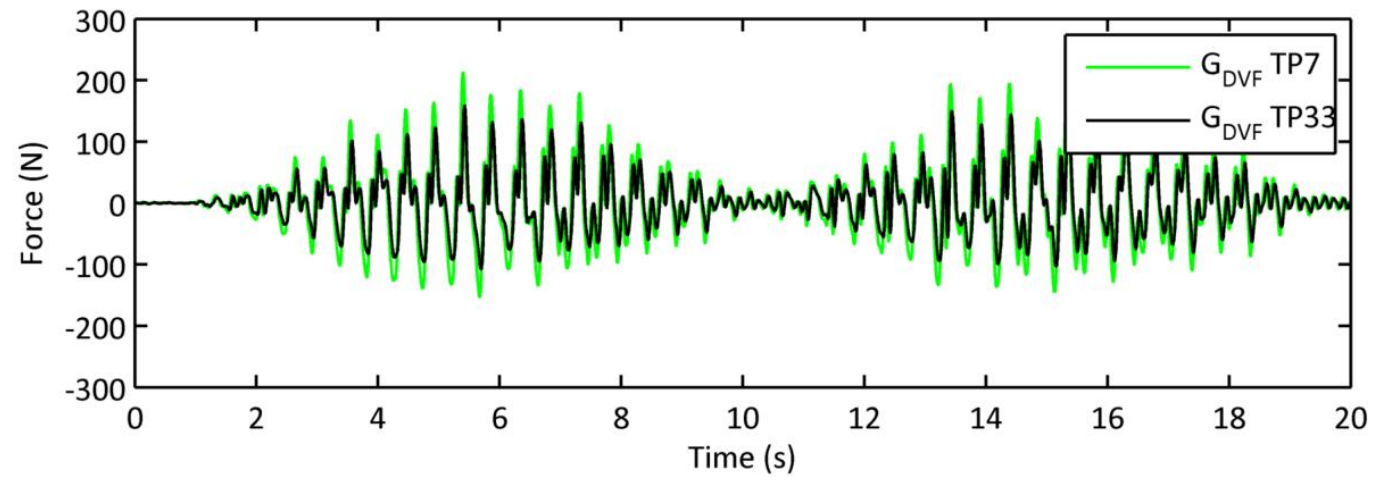

(a) Multi-SISO DVF controllers: $G_{D V F}(s)+G_{D V F}(s)$

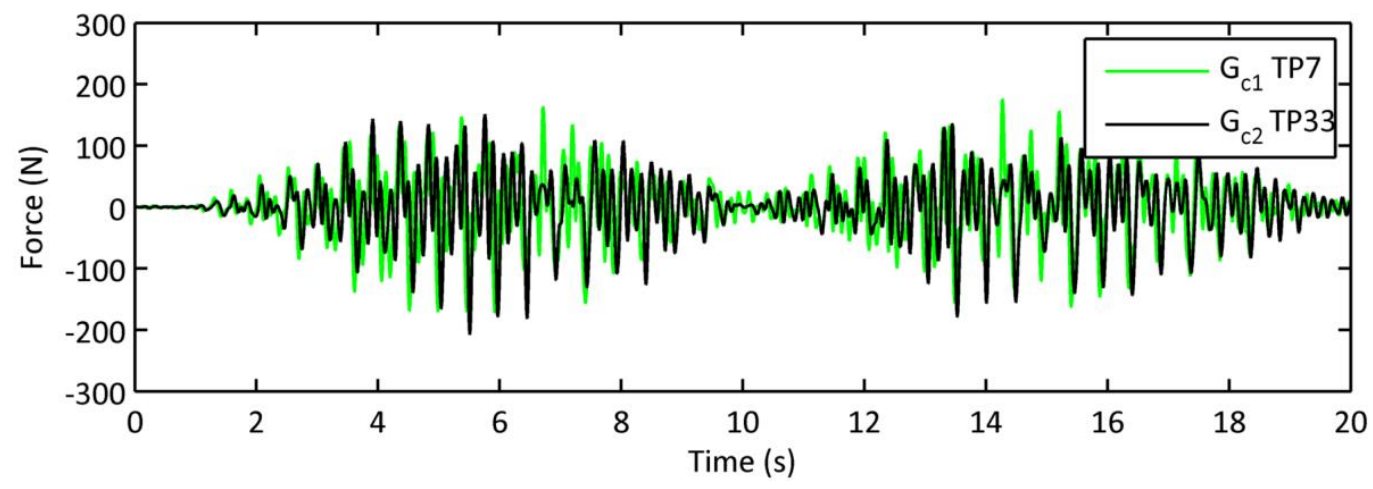

(b) Multi-SISO observer-based controllers: $G_{c 1}(s)+G_{c 2}(s)$

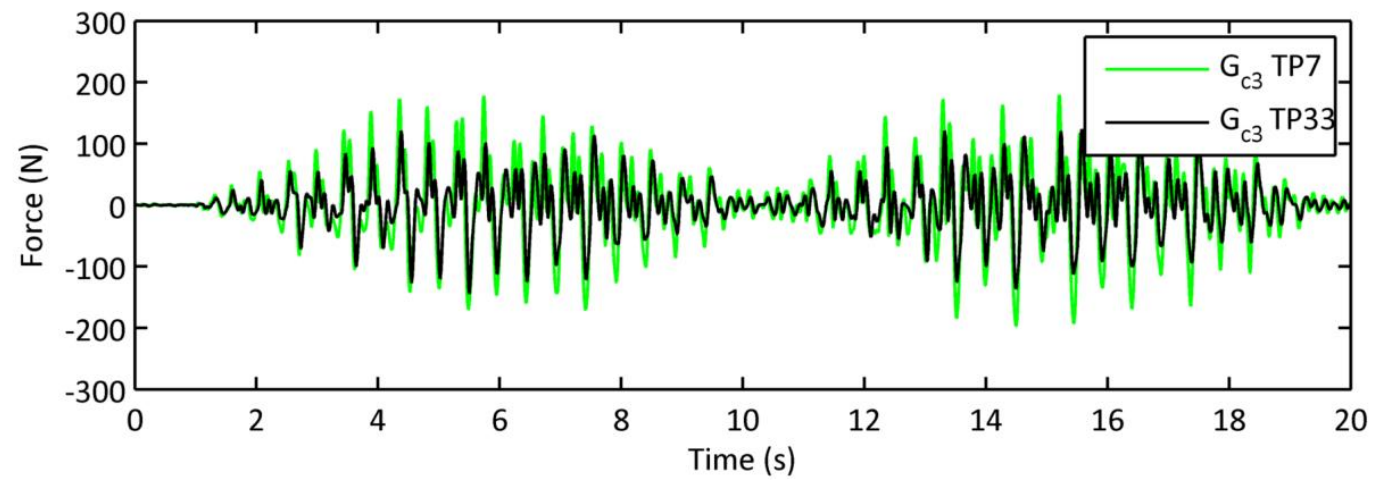

(c) Multi-SISO observer-based controllers: $G_{c 3}(s)+G_{c 3}(s)$

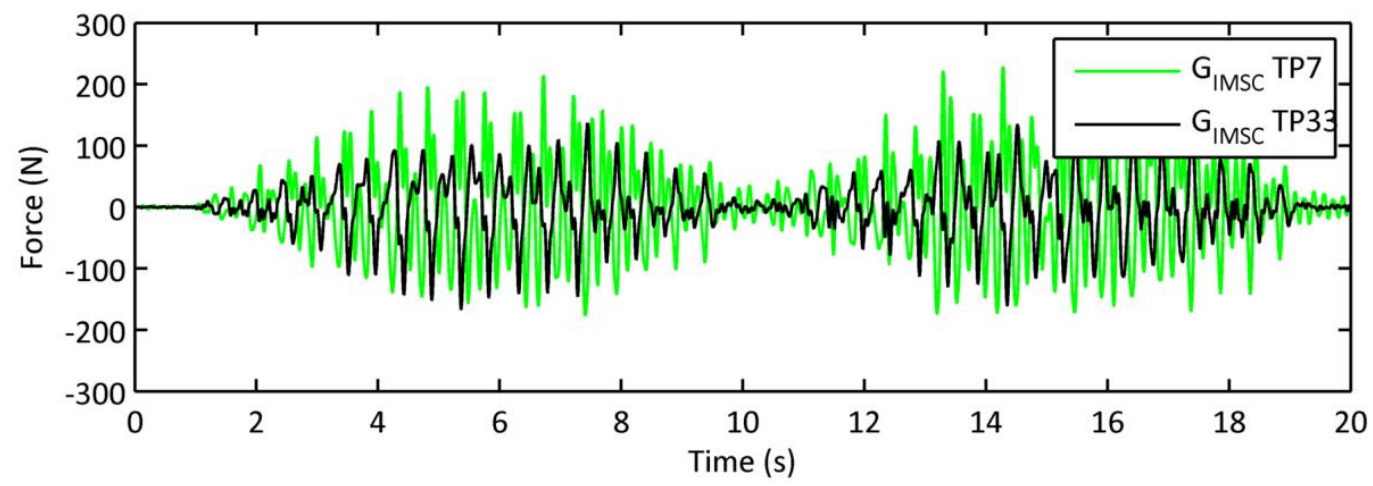

(d) Multi-SISO observer-based controllers: $\underline{G_{I M S C}}(s)$

Figure 17. Control forces for the controllers implemented in the multi-SISO and MIMO studies 
Table 6. Peak of 1s Running RMS acceleration responses for multi-SISO controller studies

\begin{tabular}{|c|c|c|c|c|}
\hline Controller & \multicolumn{2}{|c|}{$\begin{array}{c}\text { Acceleration at TP7 } \\
\left(\mathrm{m} / \mathbf{s}^{\mathbf{2}}\right)\end{array}$} & \multicolumn{2}{c|}{$\begin{array}{c}\text { Acceleration at TP33 } \\
\left(\mathrm{m} / \mathbf{s}^{\mathbf{2}}\right)\end{array}$} \\
\hline & & $\%$ reduction & & $\%$ reduction \\
\hline Uncontrolled & 0.1338 & & 0.1259 & \\
\hline$G_{D V F}(s)+G_{D V F}(s)$ & 0.0116 & 91.3 & 0.0108 & 91.4 \\
\hline$G_{C 1}(s)+G_{c 2}(s)$ & 0.0125 & 90.7 & 0.0153 & 87.8 \\
\hline$G_{C 3}(s)+G_{c 3}(s)$ & 0.0106 & 92.1 & 0.0083 & 93.4 \\
\hline$G_{I M S C}(s)$ & 0.0121 & 91.0 & 0.0116 & 90.8 \\
\hline
\end{tabular}

The results for the various controller combinations investigated in the multi-SISO and MIMO schemes in Table 6 reflect desirable attenuations in vibration responses of $85 \%-94 \%$ for all controllers. The isolation and control of the first and second vibration modes at different locations on the walkway bridge structure with observer-based controllers, $G_{c 1}(s)+G_{c 2}(s)$, in a multi-SISO scheme and the IMSC controller, $G_{I M S C}$, in a MIMO scheme still offer impressive attenuations in the global responses. This is in comparison with the DVF controller, $G_{D V F}(s)+G_{D V F}(s)$, and observer-based controller sets, $G_{c 3}(s)+G_{c 3}(s)$, implemented in multi-SISO schemes and that have been designed to offer global control functions. This work demonstrates that such an approach can still be pursued, for example, in modally dense structures in which specific problematic vibration modes can be targeted and controlled at different locations on a given structural system with the imposition of a global control objective.

Figure 17 shows the magnitudes of the control forces for all the controller sets in the multi-SISO and MIMO studies. The control forces from the controllers comprising of $G_{D V F}(s)+G_{D V F}(s)$ and $G_{c 3}(s)+G_{c 3}(s)$ are mostly in phase whilst there are phase variations with controllers for $G_{c 1}(s)+$ $G_{c 2}(s)$ and $\underline{G_{I M S C}}(s)$ as they have been designed for selective control of the lowest two vibration modes of the walkway bridge structure at different locations.

\section{Conclusions}

The studies presented here have examined various controllers designed in single-input single-output (SISO), multi-SISO and multiple-input multiple-output (MIMO) schemes. These have comprised of direct velocity feedback (DVF) controllers, observer-based controllers and an independent modal space controller (IMSC). For the multi-SISO and MIMO schemes, two actuator and sensor pairs were used.

The DVF controller is easily formulated and can be considered as a global controller in that it tackles all observable structural resonant frequencies within a frequency bandwidth considered. The observer-based controllers can be designed either for selective control or for global control objectives and are of much higher order. Their design entails a more rigorous design procedure but offers increased design freedoms that can be achieved with a single actuator and sensor pair. The approach used in the IMSC controller design relies on orthogonality of mode shapes to isolate and control specific vibration modes and requires multiple sensors and/or actuator arrays to realise various objectives. 
In the SISO controller studies, controllers with global control functions, for example, DVF and observer based controller, $G_{c 3}(s)$, offer the best vibration mitigation performances. The isolation and control of specific vibration modes, for example, as seen with observer-based controllers $G_{c 1}(s)$ and $G_{c 2}(s)$ does not necessarily present the best global vibration mitigation performances. However, at the target resonant frequencies for which they have been tuned to deal with, they do offer better performances as seen in the Fourier spectra.

The multi-SISO and MIMO controller studies present some interesting results. The desire to achieve global control through the isolation and control of target resonant frequencies at different locations on the walkway bridge structure can be achieved as demonstrated with the observer-based controllers $G_{c 1}(s)+G_{c 2}(s)$ and the IMSC controller $G_{I M S C}(s)$. This was one of the key objectives of this work. They are found to offer comparative vibration mitigation performances with the DVF controller $G_{D V F}(s)+G_{D V F}(s)$ and observer-based controllers $G_{c 3}(s)+G_{c 3}(s)$ that were originally designed for global control objectives. Based on these studies, there is a potential for the approach of isolation and control of specific vibration modes at different locations being a more beneficial procedure for dealing with modally dense structures in which particular resonant frequencies are found to be problematic.

\section{Acknowledgements}

The authors would like to acknowledge the financial assistance provided by the UK Engineering and Physical Sciences Research Council (EPSRC) through Platform Grant (Ref. EP/G061130/2) and Leadership Fellowship Grant (Ref. EP/J004081/2).

\section{References}

Bosse, A., Lim, T. W., \& Shelley, S. (2000). Modal Filters and Neural Networks for Adaptive Vibration Control. Journal of Vibration and Control, 6(4), 631-648.

BS6472-1: 2008 Guide to evaluation of human exposure to vibration in buildings. Vibration sources other than blasting, British Standards Institute, 2008.

BS6841:1987 Guide to Measurement and Evaluation of Human Exposure to Whole-Body Mechanical Vibration and Repeated Shock, British Standards Institute, 1987.

Casado, C. M., Díaz, I. M., Sebastián, J., Poncela, A. V, \& Lorenzana, A. (2013). Implementation of passive and active vibration control on an in-service footbridge. Structural Control and Health Monitoring, 20, 70-87.

Choi, S. B., \& Hedrick, J. K. (1998). An Observer-Based Controller Design Method for Improving Air / Fuel Characteristics of Spark Ignition Engines. IEEE Transactions on Automatic Control, 6(3), 325-334.

Chung, L.L., Wu, L.Y. \& Jin, T.G. (1998). Acceleration feedback control of seismic structures. Engineering Structures, 20, 62-74. 
Daley, S., Johnson, F. a., Pearson, J. B., \& Dixon, R. (2004). Active vibration control for marine applications. Control Engineering Practice, 12(4), 465-474.

Dallard, P., Fitzpatrick, A. J., Flint, A., le Bourva, S., Low, A., Ridsdill Smith, R., \& Willford, M. R. (2001). The London Millennium Footbridge. The Structural Engineer, 79(22), 17-33.

Díaz, I. M., \& Reynolds, P. (2010). Acceleration feedback control of human-induced floor vibrations. Engineering Structures, 32(1), 163-173.

Falati, S. (1999). The contribution of non-structural components to the overall dynamic behaviour of concrete floor slabs. University of Oxford.

Fang, J., Li, Q. \& Jeary, A. (2003). Modified independent modal space control of MDOF systems. Journal of Sound and Vibration, 261(3), 421-441.

González Díaz, C., \& Gardonio, P. (2007). Feedback control laws for proof-mass electrodynamic actuators. Smart Materials and Structures, 16(5), 1766-1783.

Hanagan, L. M., \& Kim, T. (2005). Preliminary Assessment for Walking-Induced Vibrations in Office Environments. Engineering Journal, First Quarter, 15-30.

Hanagan, L. M., \& Murray, T. M. (1998). Experimental Implementation of Active Control to Reduce Annoying Floor Vibrations. AISC Engineering Journal, 35(4), 123-127.

Hicks, S. J., \& Smith, A. L. (2011). Design of floor structures against human-induced vibrations. Steel Construction, 4(2), 114-120.

Highway Agency. (2002). Design manual for road and bridges: Loads for highway bridges. BD 37/01, London

Houlston, P. R., Garvey, S.D. \& Popov, A. A. (2007). Modal control of vibration in rotating machines and other generally damped systems. Journal of Sound and Vibration, 302(1-2), 104-116.

ISO 10137:2007. (2012). ISO 10137:2007 Bases for design of structures - Serviceability of buildings and walkways against vibrations, International Organization for Standardization, 2012.

ISO 2631-1 (1997) Mechanical vibration and shock - Evaluation of human exposure to whole body vibration, International Organization for Standardization, 1997.

Korondi, P. Hashimoto, H., Utkin, V. (1998). Direct Torsion Control of Flexible Shaft in an ObserverBased Discrete-Time Sliding Mode. IEEE Transactions on Industrial Electronics, 45(2), 291-296.

Liu, G. P., \& Daley, S. (1998). Stable dynamical controller design for robust polynomial pole assignment. IEE Proceedings on Control Theory Applications, 145(3), 259-264.

Liu, G. P., Duan, G. R., \& Daley, S. (2000). Stable observer-based controller design for robust statefeedback pole assignment. Proceedings of the Institution of Mechanical Engineers, Part I: Journal of Systems and Control Engineering, 214(4), 313-318.

Maciejowski, J.M. 1989, Multivariable Feedback Design, Addison-Wesley Publishing Company, ISBN 0-201-18243-2. 
Moutinho, C., Cunha, A., \& Caetano, E. (2007). Implementation of an active mass damper to control vibrations in a "lively" footbridge. III ECCOMAS Thematic Conference on Smart Structures and Materials, July 9-11.

Nyawako, D., Reynolds, P., \& Hudson, M. J. (2013). Findings with AVC design for mitigation of human induced vibrations in office floors. Topics in Dynamics of Civil Structures, Part of the series Conference Proceedings of the Society for Experimental Mechanics, 4, 37-44.

Nyawako, D. and Reynolds, P. (2015). Observer-based controller for floor vibration control with optimization algorithms, Journal of Vibration and Control, 1-16.

Preumont, a., François, a., De Man, P., \& Piefort, V. (2003). Spatial filters in structural control. Journal of Sound and Vibration, 265(1), 61-79.

Resta, F., Ripamonti, F., Cazzulani, G., \& Ferrari, M. (2010). Independent modal control for nonlinear flexible structures: An experimental test rig. Journal of Sound and Vibration, 329(8), 961-972.

Rohlfing, J., Gardonio, P., \& Thompson, D. J. (2011). Comparison of decentralized velocity feedback control for thin homogeneous and stiff sandwich panels using electrodynamic proof-mass actuators. Journal of Sound and Vibration, 330(5), 843-867.

Sandun De Silva, S., \& Thambiratnam, D. P. (2009). Dynamic characteristics of steel-deck composite floors under human-induced loads. Computers \& Structures, 87(17-18), 1067-1076.

Sepe, R. B., \& Lang, J. H. (1992). Real-Time Observer-Based (Adaptive) Control. IEEE Transactions on Automatic Control, 28(6).

Sethi, V. Song, G. (2005). Optimal Vibration Control of a Model Frame Structure Using Piezoceramic Sensors and Actuators. Journal of Vibration and Control, 11(5), 671-684.

Sethi, V. \& Song, G. (2007). Multimodal Vibration Control of a Flexible Structure using Piezoceramic Sensor and Actuator. Journal of Intelligent Material Systems and Structures, 19(5), 573-582.

Singh, S.P., Pruthi, H.S. \& Agarwal, V.P. (2003). Efficient modal control strategies for active control of vibrations. Journal of Sound and Vibration, 262, 563-575.

Vibration Serviceability Requirements For Foot And Cycle Track Bridges. (2001), BD 37/01, 72-76.

Wu, J.C., Yang, J.N. \& Schmitendorf, W.E. (1998). Acceleration feedbakc control of sesimic structures. Engineering Structures, 20(3), 222-236.

Xue, D., Chen, Y. \& Atherton, D.P. (2007). Linear feedback control: Analysis and Design with Matlab, ISBN-13: 978-0898716382.

Zilletti, M., Elliott, S. J., \& Gardonio, P. (2010). Self-tuning control systems of decentralised velocity feedback. Journal of Sound and Vibration, 329(14), 2738-2750. 